\title{
Convergence of Stochastic Interacting Particle Systems in Probability under a Sobolev Norm
}

\author{
Jian-Guo Liu \\ Dept. of Physics and Dept. of Mathematics, Duke U., USA \\ Yuan Zhang \\ Dept. of Mathematics, UCLA, USA
}

\begin{abstract}
In this paper, we consider particle systems with interaction and Brownian motion. We prove that when the initial data is from the sampling of Chorin's method, i.e., the initial vertices are on lattice points $h i \in \mathbb{R}^{d}$ with mass $\rho_{0}(h i) h^{d}$, where $\rho_{0}$ is some initial density function, then the regularized empirical measure of the interacting particle system converges in probability to the corresponding mean-field partial differential equation with initial density $\rho_{0}$, under the Sobolev norm of $L^{\infty}\left(L^{2}\right) \cap L^{2}\left(H^{1}\right)$. Our result is true for all those systems when the interacting function is bounded, Lipschitz continuous and satisfies certain regular condition. And if we further regularize the interacting particle system, it also holds for some of the most important systems of which the interacting functions are not. For systems with repulsive Coulomb interaction, this convergence holds globally on any interval $[0, t]$. And for systems with attractive Newton force as interacting function, we have convergence within the largest existence time of the regular solution of the corresponding Keller-Segel equation.
\end{abstract}

\section{Introduction}

In this paper we consider the $N$-particle system of many indistinguishable individuals interacting with each other following the same physical laws. To be specific, we consider $\left\{X_{i}(t)\right\}_{i=1}^{N} \in \mathbb{R}^{d}$ as the trajectories of the $N$ particles at time $t$. Suppose all particles have the same "weight", with certain initial data $\left\{X_{i}(0)\right\}_{i=1}^{N}$, those trajectories following the stochastic differential equations as follows:

$$
X_{i}(t)=X_{i}(0)+\frac{1}{N} \sum_{j=1}^{N} F_{0}\left(X_{i}(s)-X_{j}(s)\right) d s+\sigma B_{i}(t)
$$

where $\left\{B_{i}(t)\right\}_{i=1}^{N}$ are independent standard $d$-dimensional Brownian motions. We show that, as $N \rightarrow \infty$ and under proper assumption of the initial data, the regularized empirical measure of the interacting particle system converges in probability to the solution of the corresponding partial differential equation (PDE) as follows, which is also called the mean-field equation:

$$
\left\{\begin{array}{l}
\frac{\partial \rho}{\partial t}(x, t)=\frac{1}{2} \Delta \rho-\nabla \cdot(\rho F(x, t)) \\
F(x, t)=\int_{\mathrm{R}^{d}} F_{0}(x-y) \rho(y, t) d y .
\end{array}\right.
$$


The interest on such convergence was raised from the study of propagation of chaos, which was originated by Kac [15]. It is of interest since that to prove the propagation of chaos, one need to prove that the empirical measure of the particle system converges in law to the solution of the mean-field PDE with a proper initial condition. See the review by Sznitman [30] for reference.

Following this method, the propagation of chaos has been proved for different types of systems since the 1970s. McKean [24] proved the propagation of chaos when the interacting function $F_{0}$ is smooth. He also conjectured that when $F_{0}(x)=\delta(x)$, the one dimensional mean-field equation is the Burgers equation. This conjecture was proved [4, 13, 31]. More cases when $F_{0}$ is no longer smooth has been studied. For $d=2$ and the interacting force given by $F_{0}(x)=-\nabla^{\perp} \Phi(x)$ where $\nabla^{\perp}=\left(\frac{\partial}{\partial x_{2}}, \frac{\partial}{\partial x_{1}}\right)$ and $\Phi(x)=-\frac{1}{2} \ln |x|$, then the mean-field equation becomes the incompressible Navier-Stokes equation. When $\sigma=0$, it is the incompressible Euler equation. The mean-field limits of this type of system have been studied in [23] and [25], with or without cut-off parameters. And when $d=3$, the three dimensional NavierStokes equation and path-wise convergence rate with the stochastic vortex method have also been studied in a more recent work of [10]. And more recently, for the system with Newton/ Coulomb interaction, i.e., when $F_{0}(x)= \pm \nabla \Phi(x), \forall x \in \mathbb{R}^{d}-\{0\}$, where

$$
\Phi(x)=\left\{\begin{aligned}
-\frac{1}{2 \pi} \ln |x|, & d=2 \\
\frac{C_{d}}{|x|^{d-2}}, & d \geq 3 .
\end{aligned}\right.
$$

where $C_{d}=\frac{1}{d(d-2) \alpha_{d}}$ and $\alpha_{d}=\frac{\pi^{d / 2}}{\Gamma(d / 2+1)}$, the mean-field limit and propagation of chaos was proved by Liu and Yang, [18, 19, 20]. We refer readers to [1, 2, 11, 26, 28] for more instances of the study of propagation of chaos. And we also refer to [7] for recent progress on a blob method for the aggregation equation.

However, the mean-field limit results that the interacting particle system converges to the solution of the corresponding PDE, in the study of propagation of chaos, are usually obtained in a relatively weak sense, where the distance between two density functions are defined as Wasserstein distance. In this paper, we are, to our knowledge, for the first time to prove the convergence of the regularized empirical measure of such interacting particle system to the corresponding mean-field PDE under a stronger, Sobolev distance. Our result is generally true for all $F_{0}$ that is bounded, Lipschitz continuous and satisfies a regularity condition that will be specified in (9). And when $F_{0}$ is the Newton/ Coulomb interaction, it is also true when the interacting particle system is further regularized. For the Coulomb interaction when there is a repulsive interaction, our result remains true on any interval $[0, t]$, while when $F_{0}$ is the gradient of Newton potential, since the system now has a attractive interacting force, we have convergence within the largest existence time of the regular solution of the corresponding Keller-Segel equation.

To specify the interacting particle system we study in this paper precisely, we first need to determine the initial data. Majorly speaking, there are two ways to set up the initial data. On one hand, some previous researches like $[12,18,23,25]$ took the initial positions as independent identically distributed random variables with common density $\rho_{0}$. This approach is also known as the Monte Carlo sampling. However, this method is often inefficient in the computation. On the other hand, in [5], where Chorin first introduced the vortex method in 1973, initial positions of the vertices are assumed to be on the lattice points $h i \in \mathbb{R}^{2}$ with a weight function $\rho_{0}(h i) h^{2}$ determined by the initial density. This way of sampling has been used in [22] and more recently, in [14], and it will be the initial condition we use in this paper.

To be specific, let $\rho_{0}(x), x \in \mathbb{R}^{d}$ be the initial density that satisfies the followings: 
- $\rho_{0}(x)$ is compact supported with a compact set $D \subset \mathbb{R}^{d}$. And $\rho_{0}(x) \geq 0$ for all $x \in D$.

- $\int_{D} \rho_{0}(x) d x=1$.

- $\rho_{0}$ is a Lipschitz continuous function with Lipschitz constant $L_{\rho_{0}}$.

- $\rho_{0}(x) \in H^{k}\left(\mathbb{R}^{d}\right)$ for some $k \geq \frac{3}{2} d+2$.

For each $h>0$. Let set $\Theta_{h} \subset \mathbb{Z}^{d}$ be defined as follows:

$$
\Theta_{h}=\left\{\theta: \theta \in \mathbb{Z}^{d}, h \theta \in D\right\} .
$$

For each $\theta \in \Theta_{h}$, let

$$
C(\theta, h)=h \theta+\left[-\frac{h}{2}, \frac{h}{2}\right]^{d} .
$$

And it is east to see that $\{C(\theta, h)\}_{\theta \in \Theta_{h}}$ is a family of non-overlapping boxes and

$$
D \subset \bigcup_{\theta \in \Theta_{h}} C(\theta, h)
$$

Let $N_{h}=\operatorname{card}\left(\Theta_{h}\right)$, i.e., the number of elements in $\Theta_{h}$. Then by definition it is easy to check that

$$
L_{D}=\int_{D} 1 d x \leq h^{d} N_{h} \leq \int_{D_{1}} 1 d x=U_{D}
$$

for all $h<1$, where $D_{1}=\left\{x \in \mathbb{R}^{d}: \inf _{y \in D}|x-y|_{\infty} \leq 1\right\}$. And as $h \rightarrow \infty$, we have

$$
\lim _{h \rightarrow 0} h^{d} N_{h}=\int_{D} 1 d x
$$

[Remark]: In this paper, we will use $\|\cdot\|$ for the $L^{2}$ norm of a function or vector valued function. I.e., for any $f(x), x \in \mathbb{R}^{d}$

$$
\|f(x)\|^{2}=\int_{\mathrm{R}^{d}}|f(x)|^{2} d x
$$

and similar for the $L^{p}$ norm $\|\cdot\|_{p}$. And we will use $|\cdot|$ for the $L^{2}$ norm of a vector. I.e., for any vector $x=\left(x_{1}, x_{2}, \cdots, x_{n}\right) \in \mathbb{R}^{d}$,

$$
|x|^{2}=x_{1}^{2}+x_{2}^{2}+\cdots+x_{d}^{2}
$$

and similar for the $L^{p}$ norm $|\cdot|_{p}$.

For each $h>0$, since $\Theta_{h}$ is finite, we can have all its elements ordered under a natural ordering:

$$
\Theta_{h}=\left\{\theta_{1, h}, \theta_{2, h}, \cdots, \theta_{N_{h}, h}\right\}
$$

and have the initial point of the $i$ th particle to be $\theta_{i, h}, i=1,2, \cdots, N_{h}$.

With the initial data specified, we now formally introduce the stochastic interacting particle system in our paper:

When $F_{0}$ is bounded and Lipschitz continuous, let $\left\{X_{h, i}(t)\right\}_{i=1}^{N_{h}}$ be the interacting particle system determined by the following system of SDE:

$$
X_{h, i}(t)=\theta_{i, h} h+\int_{0}^{t}\left(\sum_{j=1}^{N_{h}} F_{0}\left(X_{h, i}(s)-X_{h, j}(s)\right) \rho_{0}\left(\theta_{j, h}\right) h^{d}\right) d s+B_{i}(t), i=1,2, \cdots, N_{h} .
$$


Noting that $F_{0}$ is bounded and Lipschitz continuous, the SDE in (5) always has a unique strong solution.

When $F_{0}$ is not bounded and Lipschitz continuous, in order to have a SDE with a unique strong solution, we need to define $\left\{X_{h, i, \delta_{h}}(t)\right\}_{i=1}^{N_{h}}$ to be the regularized interacting particle system as follows:

$$
X_{h, i, \delta_{h}}(t)=\theta_{i, h} h+\int_{0}^{t}\left(\sum_{j=1}^{N_{h}} F_{0, \delta_{h}}\left(X_{h, i, \delta_{h}}(s)-X_{h, j, \delta_{h}}(s)\right) \rho_{0}\left(\theta_{j, h}\right) h^{d}\right) d s+B_{i}(t) .
$$

where

$$
F_{0, \delta_{h}}=F_{0} * \psi_{\delta_{h}}, \quad \psi_{\delta_{h}}(x)=\delta_{h}^{-d} \psi\left(\delta_{h} x\right)
$$

and

$$
\psi(x)= \begin{cases}C(1+\cos \pi|x|)^{d+2}, & |x| \leq 1 \\ 0, & |x|>1\end{cases}
$$

with $C$ such that $\int_{\mathrm{R}^{d}} \psi(x) d x=1$. Here $\delta_{h}$ is some number goes to 0 as $h \rightarrow 0$. It will be specified later in (18).

With the (regularized) interacting particle system determined, we define the regularized empirical measure as follows: consider a function $\varphi(x) \in C_{0}^{\infty}\left(\mathbb{R}^{d}\right)$ of which the support is $\left\{|x|_{\infty} \leq 1 / 2\right\}$ such that

- $\varphi(x) \geq 0$.

- $\int_{|x|_{\infty} \leq 1} \varphi(x)=1$.

And for $\epsilon_{h}=h^{q_{0}}$ where $q_{0}$ is to be specified later in Theorem 1, let

$$
\varphi_{\epsilon_{h}}(x)=\frac{1}{\epsilon_{h}^{d}} \varphi\left(\frac{x}{\epsilon_{h}}\right) .
$$

Then for the interacting particle system when $F_{0}$ is bounded and Lipschitz continuous, the regularized empirical measure of $\left\{X_{h, i}(t)\right\}_{i=1}^{N_{h}}$ is defined as

$$
\rho_{h}(x, t)=\sum_{i=1}^{N_{h}} h^{d} \rho_{0}\left(\theta_{i, h}\right) \varphi_{\epsilon_{h}}\left(x-X_{h, i}(t)\right)
$$

And the regularized empirical measure of $\left\{X_{h, i, \delta_{h}}(t)\right\}_{i=1}^{N_{h}}$ is similarly defined as

$$
\rho_{h, \delta_{h}}(x, t)=\sum_{i=1}^{N_{h}} h^{d} \rho_{0}\left(\theta_{i, h}\right) \varphi_{\epsilon_{h}}\left(x-X_{h, i, \delta_{h}}(t)\right) .
$$

The use of the such regularized empirical measure as above is important in computation and the regularized kernel $\varphi$ is known as a blob function in the vortex method. Pioneered by Chorin in 1973 [5], the random vertex blob method is one of the most successful computational methods for fluid dynamics and other related fields. The success of the method is exemplified by the accurate computation of flow past a cylinder at the Reynolds numbers up to 9500 in the 1990s [17]. The convergence analysis for the random vortex method for the Navier-Stokes equation is given by $[12,22,23]$ in the 1980s. We refer to the book [6] for theoretical and practical use of vortex methods, refer to Goodman [12] and Long [22] for the convergence analysis of the random vortex method to the Navier-Stokes equation. We also hoped that the estimation in this paper can be adapted to do numerical analysis. 
With the regularized empirical measure defined, we need to add one more regularity condition on $F_{0}$ which assumes the existence of a constant $U_{F}<\infty$ such that

$$
\left\|F_{0}\right\|_{L^{1} \cap H^{2 d+2}\left(\mathrm{R}^{d}\right)} \leq U_{F} .
$$

Then we can have our main result of this paper, which sates that the regularized empirical measure of the interacting particle system converges to the solution of the PDE. It is presented in the following theorem:

Theorem 1. Suppose $F_{0}(x)$ is a bounded and Lipschitz continuous in $\mathbb{R}^{d}$, where the Lipschitz constant of $F_{0}(x)$ is given by $L_{F}$. And suppose that $F_{0}$ satisfies condition (9). Let $\left\{X_{h, i}(s)\right\}_{i=1}^{N_{h}}$ be the interacting particle system defined in (5) and $\rho_{h}$ be the constructed regularized empirical measure (7) with regularized parameter $\epsilon_{h}=h^{1 / 6 d}$. Let $\rho$ be the solution of the corresponding mean-field equation (2) with initial density $\rho_{0}$. Then, there is a positive function $c(t)$ (will be specified in (110)) dependent only on $t, \varphi, L_{F}, U_{F}$ and $\rho_{0}$, and a $h_{0}>0$, such that

$$
P\left(\sup _{s \in[0, t]}\left(\left\|\left(\rho-\rho_{h}\right)(\cdot, s)\right\|^{2}+\int_{0}^{s}\left\|\nabla\left(\rho-\rho_{h}\right)(\cdot, q)\right\|^{2} d q\right)<c(t) h^{1 / 12 d}\right) \geq 1-c(t) h^{1 / 12 d}
$$

for all $0<h \leq h_{0}$.

\subsection{Outline of the Proof}

Most of the rest of the paper will be devoted to the proof of Theorem 1 . The key idea of the proof is to introduce an intermediate system of self-consistent process $\hat{X}_{h, i}(t)$ defined by

$$
\hat{X}_{h, i}(t)=\theta_{i, h} h+\int_{0}^{t} F\left(\hat{X}_{h, i}(s), s\right) d s+B_{i}(t)
$$

where $\left\{B_{i}(t)\right\}_{i=1}^{N_{h}}$ are the same family of standard Brownian motions as in (5), and $F(x, t)$ is defined in (2). The first thing we note is that $F(x, t)$ is a bounded and Lipschitz function against $x$ with Lipschitz constant less than or equal to $L_{F}$. First for any $x$ and $t$,

$$
|F(x, t)| \leq \int_{\mathrm{R}^{d}}\left|F_{0}(x-y)\right| \rho(y, t) d y .
$$

Noting that $\rho$ is a probability density function on $\mathbb{R}^{d}$, we have

$$
|F(x, t)| \leq \sup _{x \in \mathrm{R}^{d}}\left|F_{0}(x)\right|<\infty .
$$

And similarly, for any $t \geq 0$ and $x_{1}, x_{2} \in \mathbb{R}^{d}$, we have

$$
\begin{aligned}
\left|F\left(x_{1}, t\right)-F\left(x_{2}, t\right)\right| & \leq \int_{\mathrm{R}^{d}}\left|F_{0}\left(x_{1}-y\right)-F_{0}\left(x_{2}-y\right)\right| \rho(y, t) d y \\
& \leq \sup _{y \in \mathrm{R}^{d}}\left|F_{0}\left(x_{1}-y\right)-F_{0}\left(x_{2}-y\right)\right| \\
& \leq\left|x_{2}-x_{1}\right| L_{F} .
\end{aligned}
$$

Thus, $\left\{\hat{X}_{h, i}(t)\right\}_{i=1}^{N_{h}}$ is a family of independent strong solutions the same SDE with the same initial values as the interacting particle system. Then consider the similar regularized empirical measure:

$$
\hat{\rho}_{h}(x, t)=\sum_{i=1}^{N_{h}} h^{d} \rho_{0}\left(\theta_{i, h}\right) \varphi_{\epsilon_{h}}\left(x-\hat{X}_{h, i}(t)\right) .
$$


If we can estimate both the distances between $\rho_{h}(x, t)$ and $\hat{\rho}_{h}(x, t)$, and the distance between $\hat{\rho}_{h}(x, t)$ and $\rho(x, t)$, we will be able to prove Theorem 1 .

\section{Control the Distance Between $\rho_{h}(x, t)$ and $\hat{\rho}_{h}(x, t)$}

To estimate the distance between the regularized empirical measure constructed from the interacting particle system and that constructed from the self-consistent process, we use a recent proved result by Huang and Liu [14] that estimates the $l_{h}^{p}$ norm of $\hat{X}_{h, i}(t)-X_{h, i}(t)$. For any vector $\vec{x}=\left(x_{1}, x_{2}, \cdots, x_{N_{h}}\right)$ and $p \geq 1$, its $l_{h}^{p}$ norm is defined as

$$
|\vec{x}|_{l_{h}^{p}}=\left(h^{d} \sum_{i=1}^{N_{h}}\left|x_{i}\right|^{p}\right)^{\frac{1}{p}} .
$$

According to Theorem 6.1 in [14], there exist a $p>1$ such that for all $0<h \leq h_{0}$ with $h_{0}$ sufficiently small, there exists two positive constants $C$ and $C^{\prime}$ depending on $t, p, d, U_{F}, \rho_{0}$ and the diameter of $D$. The following estimate holds true:

$$
P\left(\max _{0 \leq s \leq t}\left|\hat{X}_{h, i}(s)-X_{h, i}(s)\right|_{l_{h}^{p}}<\Lambda h|\ln h|\right) \geq 1-h^{C \Lambda|\ln h|}
$$

for all $\Lambda \geq C^{\prime}$. Then under the high probability event

$$
E_{h}=\left\{\max _{0 \leq s \leq t}\left|\hat{X}_{h, i}(s)-X_{h, i}(s)\right|_{l_{h}^{p}}<C^{\prime} h|\ln h|\right\}
$$

we can use the $l_{h}^{p}$ norm of $\hat{X}_{h, i}(t)-X_{h, i}(t)$ to estimate the distance between the empirical measures $\rho_{h}(x, t)$ and $\hat{\rho}_{h}(x, t)$. Details of this part can be found in Section 2.

\section{Control the Distance Between $\rho(x, t)$ and $\hat{\rho}_{h}(x, t)$}

In this second step we estimate the distance between the empirical measure $\hat{\rho}_{h}(x, t)$ constructed from the self-consistent process and the solution of the PDE. To estimate this distance, we have a theorem as follows:

Theorem 2. Let $\left\{\hat{X}_{h, i}(t)\right\}_{i=1}^{N_{h}}$ be the self-consistent system and $\hat{\rho}_{h}(x, t)$ be the constructed regularized empirical measure with regularized parameter $\epsilon_{h}=h^{1 / 6 d}$. Let $\rho$ be the solution of the corresponding mead field equation (2) with initial density $\rho_{0}$. Then, there is a positive function $c_{1}(t), t>0$ (will be specified in (109)) dependent only on $t, \varphi$, and $\rho_{0}$, such that

$$
P\left(\sup _{s \in[0, t]}\left(\left\|\left(\rho-\hat{\rho}_{h}\right)(\cdot, s)\right\|^{2}+\int_{0}^{s}\left\|\nabla\left(\rho-\hat{\rho}_{h}\right)(\cdot, q)\right\|^{2} d q\right) \leq c_{1}(t) h^{1 / 12 d}\right) \geq 1-c_{1}(t) h^{1 / 12 d} .
$$

where $C_{0}=2 d L_{F}$ and $L_{F}$ is the Lipschitz constant of $F_{0}$.

The proof of Theorem 2 is similar to the one reported recently in the authors' conference note [21], where some preliminary work of this paper is reported with a much simplified system with only drift and diffusion but no interactions. In that case, the mean-field PDE is the Fokker-Planck equation and there are no mass function on each data points in the empirical measure. Here we generalized the proof and make it adapted to the new definition of empirical measure in this paper and to the self-consistent system. To prove this theorem, we take the following steps: 
(1) First we use Ito's formula to decompose the $L^{\infty}\left(L^{2}\right) \cap L^{2}\left(H^{1}\right)$ norm of the difference between $\rho(x, t)$ and $\hat{\rho}_{h}(x, t)$. Here we will have a term that is from the free energy estimation of PDE, a term of initial error, a term of truncation error, and a term of martingale error. Details can be found in Section 3.

(2) Second, we prove a proposition on the separation of the self-consistent system which shows that for the self-consistent system $\left\{\hat{X}_{h, i}(t)\right\}_{i=1}^{N_{h}}$, there cannot be too many particles stay too close with each other. To prove this separation problem, we use Girsanov Theorem to reduce it to a separation problem of standard Brownian motions. The proof of the Brownian motion case is technical, where cases for $d=2, d=2$ or $d \geq 3$ will be proved differently. Details can be found in Section 4.

(3) Then we estimate the term of truncation error. We are able to use the result we proved in the proposition of separation and the fact that $\varphi_{\epsilon_{h}}$ is supported on $\left\{x:|x|_{\infty}<\epsilon_{h}\right\}$ to bounded the truncation error under a high probability event. Details can be found in Section 5 .

(4) Since the empirical measure is rescaled by $h^{d}$, we can use standard stochastic differential equation argument to estimate the martingale errors in the estimation. Details can be found in Section 6.

(5) Note that $\varphi \in C_{0}^{\infty}$ and that the initial density $\rho_{0}$ is Lipschitz continuous. We can estimate the initial error using standard calculations. Details can be found in Section 7.

(6) After we have estimated the initial, truncation and the martingale errors, we can use Gronwall's inequality to estimate the distance between the empirical measure $\hat{\rho}_{h}(x, t)$ and the solution of the PDE and finish the proof. Details can be found in Section 8 .

Combining Part I and Part II, we finish the proof of Theorem 1.

\subsection{Newton and Coulomb Interactions}

With Theorem 1 holds true, when the interacting function $F_{0}$ is not bounded and Lipschitz continuous, this convergence result may remain hold. The intuition behind this generalization is that, though $F_{0}$ itself is not bounded and Lipschitz continuous, the function $F$ defined in (2) may still be bounded and Lipschitz continuous (in a certain interval). Thus the SDE of self-consistent system in (11) is still well defined and has a unique strong solution. Note that the proof of Theorem 2 depends only on the fact that $F$ rather than $F_{0}$ is bounded and Lipschitz continuous against $x$. We are still able to estimate the distance between the regularized empirical measure $\hat{\rho}_{h}(x, t)$ of the self-consistent system, and the solution of the PDE.

Thus, to show the convergence result in Theorem 1, it suffices to estimate the distance between the regularized empirical measure $\hat{\rho}_{h}(x, t)$ of the self-consistent system and the regularized empirical measure $\rho_{h, \delta_{h}}(x, t)$ of the regularized interacting particle system. Fortunately, the results recently proved in [14] give us exactly the same estimation as in (14), between of the $l_{h}^{p}$ distance between $\hat{X}_{h, i}(s)$ and $X_{h, i, \delta_{h}}(s)$, when the function $F_{0}$ is Coulomb or Newton Interactions. Thus exactly the same argument as in Section 2 will finish the proof for those systems.

Newton Interaction. In this case, the aggregation function is given by $F_{0}(x)=\nabla \Phi(x)$, $\forall x \in \mathbb{R}^{d}-\{0\}$, where

$$
\Phi(x)=\left\{\begin{aligned}
-\frac{1}{2 \pi} \ln |x|, & d=2 \\
\frac{C_{d}}{|x|^{d-2}}, & d \geq 3 .
\end{aligned}\right.
$$


And the mean-field PDE is the Keller-Segel equation. Noting that $\rho_{0}(x) \in H^{k}\left(\mathbb{R}^{d}\right)$ for some $k \geq \frac{3}{2} d+1$, this implies the existence of the unique local solution to the Keller-Segel equation with the follow regularities

$$
\|\rho\|_{L^{\infty}\left(0, T, H^{k}\left(\mathrm{R}^{d}\right)\right)} \leq C\left(\left\|\rho_{0}\right\|_{H^{k}\left(\mathrm{R}^{d}\right)}\right)
$$

and

$$
\left\|\partial_{t} \rho\right\|_{L^{\infty}\left(0, T, H^{k-2}\left(\mathrm{R}^{d}\right)\right)} \leq C\left(\left\|\rho_{0}\right\|_{H^{k}\left(\mathrm{R}^{d}\right)}\right)
$$

where $T>0$ depends only on $\left\|\rho_{0}\right\|_{H^{k}\left(\mathrm{R}^{d}\right)}$. Denote $T_{\max }$ to be the largest existence time such that (16) and (17) is valid. According to Sobolev imbedding theorem, one has $\rho(x, t) \in$ $C^{k-d / 2-1}$ for any $t \in[0, T]$. And for

$$
F(x, t)=\int_{\mathrm{R}^{d}} F_{0}(x-y) \rho(y, t) d y
$$

using the Sobolev imbedding theorem again gives us

$$
\|F\|_{L^{\infty}\left(0, T, W^{k-d / 2-2, \infty}\left(\mathrm{R}^{d}\right)\right)} \leq C\|F\|_{L^{\infty}\left(0, T, H^{k+1}\left(\mathrm{R}^{d}\right)\right)} \leq C\left(\left\|\rho_{0}\right\|_{H^{k}\left(\mathrm{R}^{d}\right)}\right)
$$

and

$$
\left\|\partial_{t} F\right\|_{L^{\infty}\left(0, T, W^{\left.k-d / 2-2, \infty\left(\mathrm{R}^{d}\right)\right)}\right.} \leq C\left(\left\|\rho_{0}\right\|_{H^{k}\left(\mathrm{R}^{d}\right)}\right) .
$$

Thus for any $T<T_{\max }, F(x, s)$ is a bounded and Lipschitz continuous on $\mathbb{R}^{d} \times[0, T]$, with Lipschitz constants uniformly bounded. Thus, Theorem 2 in this paper holds for the regularized empirical measure $\hat{\rho}_{h}(x, t)$ of the self-consistent system, and the solution of the corresponding Keller-Segel equation.

With the distance between the self-consistent system and the mean-field PDE estimated, let

$$
\delta_{h}=h^{\kappa}
$$

where $\kappa \in(1 / 2,1)$. Then according to Theorem 1.1 in [14], we have for $p>d /(1-\kappa)$, and $h$ sufficiently small, there exists two positive constants $C$ and $C^{\prime}$ depending on $T_{\max }, p, d$ and $\rho_{0}$ and the diameter of $D$. The following estimate holds true:

$$
P\left(\max _{0 \leq s \leq t}\left|\hat{X}_{h, i}(s)-X_{h, i}(s)\right|_{l_{h}^{p}}<\Lambda h|\ln h|\right) \geq 1-h^{C \Lambda|\ln h|}
$$

for all $\Lambda \geq C^{\prime}$. Noting that the inequality above has the same form as (14), then the argument in Section 2 gives the estimation between $\hat{X}_{h, i}(s)$ and $X_{h, i, \delta_{h}}(s)$ and gives us the following corollary on the convergence of system with Newton interaction:

Corollary 1. For any $t<T_{\max }$, suppose $F_{0}(x)$ is given by the Newton Interaction. Let $\left\{X_{h, i, \delta_{h}}(s)\right\}_{i=1}^{N_{h}}$ be the regularized interacting particle system defined in (6) with $\delta_{h}$ defined in (18), and $\rho_{h, \delta_{h}}$ be the constructed regularized empirical measure (8) with regularized parameter $\epsilon_{h}=h^{1 / 6 d}$. Let $\rho$ be the solution of the corresponding Keller-Segel equation with initial density $\rho_{0}$. Then, there is a positive function $c(t)$ (will be specified in (110)) dependent only on $t, \varphi$, $L_{F}$ and $\left\|\rho_{0}\right\|$, and $a h_{0}>0$, such that

$$
P\left(\sup _{s \in[0, t]}\left(\left\|\left(\rho-\rho_{h, \delta_{h}}\right)(\cdot, s)\right\|^{2}+\int_{0}^{s}\left\|\nabla\left(\rho-\rho_{h, \delta_{h}}\right)(\cdot, q)\right\|^{2} d q\right)<c(t) h^{1 / 12 d}\right) \geq 1-c(t) h^{1 / 12 d}
$$

for all $0<h \leq h_{0}$. 
Coulomb Interaction. In this case, the interaction function is given by $F_{0}(x)=-\nabla \Phi(x)$, $\forall x \in \mathbb{R}^{d}-\{0\}$, where

$$
\Phi(x)=\left\{\begin{aligned}
-\frac{1}{2 \pi} \ln |x|, & d=2 \\
\frac{C_{d}}{|x|^{d-2}}, & d \geq 3 .
\end{aligned}\right.
$$

And the mean-field PDE is the drift-diffusion equation. Thus again let $T_{\max }$ be the same largest existence time of a regular solution. According to [20], $T_{\max }=\infty$ So again using Sobolev embedding theorem on

$$
F(x, t)=\int_{\mathrm{R}^{d}} F_{0}(x-y) \rho(y, t) d x
$$

we have that for any $t>0, F(x, s)$ is bounded and Lipschitz continuous on $\mathbb{R}^{d} \times[0, t]$, with Lipschitz constants uniformly bounded. Thus, Theorem 2 in this paper holds for the regularized empirical measure $\hat{\rho}_{h}(x, t)$ of the self-consistent system, and the solution of the corresponding drift-diffusion equation.

Moreover, according to exactly the same argument, see Remark 1.1 in [14], let $\delta_{h}$ be the same as defined in (18), there is a $p>d /(1-\kappa)$, and $h$ sufficiently small, there exists two positive constants $C$ and $C^{\prime}$ depending on $T_{\max }, p, d$ and $\rho_{0}$ and the diameter of $D$. The following estimate holds true:

$$
P\left(\max _{0 \leq s \leq t}\left|\hat{X}_{h, i}(s)-X_{h, i}(s)\right|_{l_{h}^{p}}<\Lambda h|\ln h|\right) \geq 1-h^{C \Lambda|\ln h|}
$$

for all $\Lambda \geq C^{\prime}$. Thus we have the following corollary on the convergence of system with Coulomb interaction:

Corollary 2. For any $t>0$, suppose $F_{0}(x, s)$ is given by the Coulomb Interaction. Let $\left\{X_{h, i, \delta_{h}}(s)\right\}_{i=1}^{N_{h}}$ be the regularized interacting particle system defined in (6) with $\delta_{h}$ defined in (18), and $\rho_{h, \delta_{h}}$ be the constructed regularized empirical measure (8) with regularized parameter $\epsilon_{h}=h^{1 / 6 d}$. Let $\rho$ be the solution of the corresponding drift-diffusion equation with initial density $\rho_{0}$. Then, there is a positive function $c(t)$ (will be specified in (110)) dependent only on $t, \varphi, L_{F}$ and $\left\|\rho_{0}\right\|$, and $a h_{0}>0$, such that

$$
P\left(\sup _{s \in[0, t]}\left(\left\|\left(\rho-\rho_{h, \delta_{h}}\right)(\cdot, s)\right\|^{2}+\int_{0}^{s}\left\|\nabla\left(\rho-\rho_{h, \delta_{h}}\right)(\cdot, q)\right\|^{2} d q\right)<c(t) h^{1 / 12 d}\right) \geq 1-c(t) h^{1 / 12 d}
$$

for all $0<h \leq h_{0}$.

\section{The $L^{\infty}\left(L^{2}\right) \cap L^{2}\left(H^{1}\right)$ Distance between $\rho_{h}(x, t)$ and $\hat{\rho}_{h}(x, t)$}

According to Theorem 6.1 in [14], it has been proved that let $\left\{X_{h, i}(t)\right\}_{i=1}^{N_{h}}$ and $\left\{\hat{X}_{h, i}(t)\right\}_{i=1}^{N_{h}}$ be the original interacting particle system and the self-consistent system with initial values of $\left\{\theta_{i, h} h\right\}_{i=1}^{N_{h}}$, which are specified in (5) and (11), then there exist a $p>1$ such that for all $0<h \leq h_{0}$ with $h_{0}$ sufficiently small, there exists two positive constants $C$ and $C^{\prime}$ depending on $t, p, d$ and $\rho_{0}$ and the diameter of $D$. The following estimate holds true for all $\Lambda>C^{\prime}$ :

$$
P\left(\max _{0 \leq s \leq t}\left|\hat{X}_{h, i}(s)-X_{h, i}(s)\right|_{l_{h}^{p}}<\Lambda h|\ln h|\right) \geq 1-h^{C \Lambda|\ln h|}
$$


where the $l_{h}^{p}$ norm is defined in (13). Then under the high probability event

$$
E_{h}=\left\{\max _{0 \leq s \leq t}\left|\hat{X}_{h, i}(s)-X_{h, i}(s)\right|_{l_{h}^{p}}<C^{\prime} h|\ln h|\right\},
$$

we will use the estimation of the distance between $\left\{X_{h, i}(s)\right\}_{i=1}^{N_{h}}$ and $\left\{\hat{X}_{h, i}(s)\right\}_{i=1}^{N_{h}}$ to estimate the distance between the two empirical measures constructed from them. We have the theorem as follows:

Theorem 3. Let $\left\{X_{h, i}(t)\right\}_{i=1}^{N_{h}}$ be the interacting particle system defined in (5) and $\left\{\hat{X}_{h, i}(t)\right\}_{i=1}^{N_{h}}$ be the self-consistent system defined in (11). $\rho_{h}(x, t)$ and $\hat{\rho}_{h}(x, t)$ be the constructed regularized empirical measure respectively, with regularized parameter $\epsilon_{h}=h^{1 / 6 d}$. Then, there is a positive function $c_{0}(t), t>0$ (will be specified in (31)) dependent only on $t, \varphi, U_{F}, L_{F}$, and $\rho_{0}$, such that

$$
P\left(\sup _{s \in[0, t]}\left(\left\|\left(\rho_{h}-\hat{\rho}_{h}\right)(\cdot, s)\right\|^{2}+\int_{0}^{s}\left\|\nabla\left(\rho_{h}-\hat{\rho}_{h}\right)(\cdot, q)\right\|^{2} d q\right)<c_{0}(t) h^{1 / 12 d}\right) \geq 1-c_{0}(t) h^{1 / 12 d} .
$$

Proof. Recall that

$$
\rho_{h}(x, s)=\sum_{i=1}^{N_{h}} h^{d} \rho_{0}\left(\theta_{i, h}\right) \varphi_{\epsilon_{h}}\left(x-X_{h, i}(s)\right)
$$

and

$$
\hat{\rho}_{h}(x, s)=\sum_{i=1}^{N_{h}} h^{d} \rho_{0}\left(\theta_{i, h}\right) \varphi_{\epsilon_{h}}\left(x-\hat{X}_{h, i}(s)\right) .
$$

Then for any $s \in[0, t]$, the $L^{2}$ norm of the difference is given by

$$
\begin{aligned}
\left\|\rho_{h}(x, s)-\hat{\rho}_{h}(x, s)\right\| & \leq \sum_{i=1}^{N_{h}} h^{d} \rho_{0}\left(\theta_{i, h}\right)\left\|\varphi_{\epsilon_{h}}\left(x-X_{h, i}(s)\right)-\varphi_{\epsilon_{h}}\left(x-\hat{X}_{h, i}(s)\right)\right\| \\
& =\sum_{i=1}^{N_{h}} h^{d} \epsilon_{h}^{-d} \rho_{0}\left(\theta_{i, h}\right)\left\|\varphi\left(\frac{x-X_{h, i}(s)}{\epsilon_{h}}\right)-\varphi\left(\frac{x-\hat{X}_{h, i}(s)}{\epsilon_{h}}\right)\right\| .
\end{aligned}
$$

Note that since $\varphi \in C_{0}^{\infty}$, for any $i$, according to mid-value theorem

$$
\left|\varphi\left(\frac{x-X_{h, i}(s)}{\epsilon_{h}}\right)-\varphi\left(\frac{x-\hat{X}_{h, i}(s)}{\epsilon_{h}}\right)\right| \leq c_{d}\left|\frac{X_{h, i}(s)-\hat{X}_{h, i}(s)}{\epsilon_{h}}\right| \max _{k=1, \cdots, d}\left\|\frac{\partial \varphi}{\partial x_{k}}\right\|_{\infty}
$$

where $c_{d}$ is some constant that depends only on $d$, and that

$$
\left|\varphi\left(\frac{x-X_{h, i}(s)}{\epsilon_{h}}\right)-\varphi\left(\frac{x-\hat{X}_{h, i}(s)}{\epsilon_{h}}\right)\right| \equiv 0
$$

for all $x \notin U_{i}=\left\{y:\left|y-X_{h, i}\right| \leq \epsilon_{h} / 2\right\} \cup\left\{y:\left|y-\hat{X}_{h, i}\right| \leq \epsilon_{h} / 2\right\}$. Thus

$$
\begin{aligned}
\left\|\varphi\left(\frac{x-X_{h, i}(s)}{\epsilon_{h}}\right)-\varphi\left(\frac{x-\hat{X}_{h, i}(s)}{\epsilon_{h}}\right)\right\| & \leq c_{d}\left|\frac{X_{h, i}(s)-\hat{X}_{h, i}(s)}{\epsilon_{h}}\right| \max _{k=1, \cdots, d}\left\|\frac{\partial \varphi}{\partial x_{k}}\right\|_{\infty} \sqrt{\int_{U_{i}} d x} \\
& \leq 2 c_{d}\left|\frac{X_{h, i}(s)-\hat{X}_{h, i}(s)}{\epsilon_{h}}\right| \max _{k=1, \cdots, d}\left\|\frac{\partial \varphi}{\partial x_{k}}\right\|_{\infty} \epsilon_{h}^{d / 2} .
\end{aligned}
$$


Plugging (23) in (22) and noting that $\rho_{0}$ is Lipschitz continuous and thus bounded, we have

$$
\left\|\rho_{h}(x, s)-\hat{\rho}_{h}(x, s)\right\| \leq 2 c_{d}\left\|\rho_{0}\right\|_{\infty} \max _{k=1, \cdots, d}\left\|\frac{\partial \varphi}{\partial x_{k}}\right\|_{\infty} \epsilon_{h}^{-d / 2-1}\left|\hat{X}_{h, i}(s)-X_{h, i}(s)\right|_{l_{h}^{1}}
$$

Noting that according to Jensen's inequality, for any $\vec{x} \in \mathbb{R}^{N_{h}}$ and $p \geq 1$ we always have

$$
\left(\frac{\sum_{i=1}^{N_{h}}\left|x_{i}\right|^{p}}{N_{h}}\right)^{\frac{1}{p}} \geq \frac{\sum_{i=1}^{N_{h}}\left|x_{i}\right|}{N_{h}}
$$

Combining this with (4), we have

$$
\begin{aligned}
\left|\hat{X}_{h, i}(s)-X_{h, i}(s)\right|_{l_{h}^{1}} & \leq\left(h^{d} N_{h}\right)^{1-p^{-1}}\left|\hat{X}_{h, i}(s)-X_{h, i}(s)\right|_{l_{h}^{p}} \\
& \leq U_{D}\left|\hat{X}_{h, i}(s)-X_{h, i}(s)\right|_{l_{h}^{p}} .
\end{aligned}
$$

Plugging this inequality to (24) and according to the definition of $E_{h}$, we have under event $E_{h}$ :

$$
\begin{aligned}
\left\|\rho_{h}(x, s)-\hat{\rho}_{h}(x, s)\right\| & \leq 2 c_{d}\left\|\rho_{0}\right\|_{\infty} \max _{k=1, \cdots, d}\left\|\frac{\partial \varphi}{\partial x_{k}}\right\|_{\infty} \epsilon_{h}^{-d / 2-1} U_{D} C^{\prime} h|\ln h| \\
& \leq 2 c_{d}\left\|\rho_{0}\right\|_{\infty} \max _{k=1, \cdots, d}\left\|\frac{\partial \varphi}{\partial x_{k}}\right\|_{\infty} \epsilon_{h}^{-d / 2-1} U_{D} C^{\prime} h^{2 / 3}
\end{aligned}
$$

when $h$ is sufficiently small. Noting that $\epsilon_{h}=h^{1 / 6 d}$, we have

$$
\epsilon_{h}^{-d / 2-1} h^{2 / 3}=h^{7 / 12-1 / 6 d}<h^{1 / 12 d} .
$$

Combining this observation with the fact that (26) holds true for all $s \in[0, t]$, we have

$$
\sup _{s \in[0, t]}\left\|\rho_{h}(x, s)-\hat{\rho}_{h}(x, s)\right\| \leq 2 c_{d}\left\|\rho_{0}\right\|_{\infty} \max _{k=1, \cdots, d}\left\|\frac{\partial \varphi}{\partial x_{k}}\right\|_{\infty} U_{D} C^{\prime} h^{1 / 6 d} .
$$

Then similarly for any $s \in[0, t]$ consider

$$
\left\|\nabla \rho_{h}(x, s)-\nabla \hat{\rho}_{h}(x, s)\right\| \leq \sum_{k=1}^{d}\left\|\frac{\partial \rho_{h}(x, s)}{\partial x_{k}}-\frac{\partial \hat{\rho}_{h}(x, s)}{\partial x_{k}}\right\| .
$$

Then for each $i$ we can similarly we have

$$
\begin{aligned}
\left\|\frac{\partial \rho_{h}(x, s)}{\partial x_{k}}-\frac{\partial \hat{\rho}_{h}(x, s)}{\partial x_{k}}\right\| & \leq \sum_{i=1}^{N_{h}} h^{d} \rho_{0}\left(\theta_{i, h}\right)\left\|\frac{\partial \varphi_{\epsilon_{h}}\left(x-X_{h, i}(s)\right)}{\partial x_{k}}-\frac{\partial \varphi_{\epsilon_{h}}\left(x-\hat{X}_{h, i}(s)\right)}{\partial x_{k}}\right\| \\
& =\sum_{i=1}^{N_{h}} h^{d} \epsilon_{h}^{-d-1} \rho_{0}\left(\theta_{i, h}\right)\left\|\frac{\partial \varphi}{\partial x_{k}}\left(\frac{x-X_{h, i}(s)}{\epsilon_{h}}\right)-\frac{\partial \varphi}{\partial x_{k}}\left(\frac{x-\hat{X}_{h, i}(s)}{\epsilon_{h}}\right)\right\| .
\end{aligned}
$$

Again according to mid-value theorem, we have

$$
\left|\frac{\partial \varphi}{\partial x_{k}}\left(\frac{x-X_{h, i}(s)}{\epsilon_{h}}\right)-\frac{\partial \varphi}{\partial x_{k}}\left(\frac{x-\hat{X}_{h, i}(s)}{\epsilon_{h}}\right)\right| \leq c_{d}\left|\frac{X_{h, i}(s)-\hat{X}_{h, i}(s)}{\epsilon_{h}}\right| \max _{j, k=1, \cdots, d}\left\|\frac{\partial^{2} \varphi}{\partial x_{j} \partial x_{k}}\right\|_{\infty}
$$

and

$$
\left|\frac{\partial \varphi}{\partial x_{k}}\left(\frac{x-X_{h, i}(s)}{\epsilon_{h}}\right)-\frac{\partial \varphi}{\partial x_{k}}\left(\frac{x-\hat{X}_{h, i}(s)}{\epsilon_{h}}\right)\right| \equiv 0
$$


for all $x \notin U_{i}=\left\{y:\left|y-X_{h, i}\right| \leq \epsilon_{h} / 2\right\} \cup\left\{y:\left|y-\hat{X}_{h, i}\right| \leq \epsilon_{h} / 2\right\}$. Thus

$$
\left\|\frac{\partial \varphi}{\partial x_{k}}\left(\frac{x-X_{h, i}(s)}{\epsilon_{h}}\right)-\frac{\partial \varphi}{\partial x_{k}}\left(\frac{x-\hat{X}_{h, i}(s)}{\epsilon_{h}}\right)\right\| \leq 2 c_{d}\left|\frac{X_{h, i}(s)-\hat{X}_{h, i}(s)}{\epsilon_{h}}\right| \max _{j, k=1, \cdots, d}\left\|\frac{\partial^{2} \varphi}{\partial x_{j} \partial x_{k}}\right\|_{\infty} \epsilon_{h}^{d / 2}
$$

which implies that

$$
\left\|\frac{\partial \rho_{h}(x, s)}{\partial x_{k}}-\frac{\partial \hat{\rho}_{h}(x, s)}{\partial x_{k}}\right\| \leq 2 c_{d}\left\|\rho_{0}\right\|_{\infty} \max _{j, k=1, \cdots, d}\left\|\frac{\partial^{2} \varphi}{\partial x_{j} \partial x_{k}}\right\|_{\infty} \epsilon_{h}^{-d / 2-2} U_{D} C^{\prime} h^{2 / 3}
$$

when $h$ is sufficiently small. Again noting that $\epsilon_{h}=h^{1 / 6 d}$, we have

$$
\epsilon_{h}^{-d / 2-2} h^{2 / 3}=h^{7 / 12-1 / 3 d}<h^{1 / 12 d} .
$$

And note that (28) holds for all $k=1,2, \cdots, d$. Thus for any $s \in[0, t]$,

$$
\left\|\nabla \rho_{h}(x, s)-\nabla \hat{\rho}_{h}(x, s)\right\| \leq 2 d c_{d}\left\|\rho_{0}\right\|_{\infty} \max _{j, k=1, \cdots, d}\left\|\frac{\partial^{2} \varphi}{\partial x_{j} \partial x_{k}}\right\|_{\infty} U_{D} C^{\prime} h^{1 / 6 d}
$$

and

$$
\int_{s=0}^{t}\left\|\nabla \rho_{h}(x, s)-\nabla \hat{\rho}_{h}(x, s)\right\| d s \leq 2 d t c_{d}\left\|\rho_{0}\right\|_{\infty} \max _{j, k=1, \cdots, d}\left\|\frac{\partial^{2} \varphi}{\partial x_{j} \partial x_{k}}\right\|_{\infty} U_{D} C^{\prime} h^{1 / 6 d} .
$$

Let

$$
c_{0}(t)=2 d t c_{d}\left\|\rho_{0}\right\|_{\infty} \max _{j, k=1, \cdots, d}\left\|\frac{\partial^{2} \varphi}{\partial x_{j} \partial x_{k}}\right\|_{\infty} U_{D} C^{\prime}+2 c_{d}\left\|\rho_{0}\right\|_{\infty} \max _{k=1, \cdots, d}\left\|\frac{\partial \varphi}{\partial x_{k}}\right\|_{\infty} U_{D} C^{\prime}+1 .
$$

It is easy to see that

$$
h^{\Lambda C^{\prime}|\ln h|}<h^{1 / 12 d}<h^{1 / 12 d} c_{0}(t)
$$

when $h$ is sufficiently small. Thus the proof is complete.

\section{Decomposition of Errors}

Since we have estimated the distance between the empirical measures constructed from the interacting particle system and the self-consistent system. The remainder of the paper will mostly devote to the proof of Theorem 2. First, as described in the outline of the proof, we use Ito's formula to separate this distance into a term of the free energy estimation of PDE, a term of initial error, a truncation error and a martingale error. To be precise, we have a proposition as follows:

Proposition 1. For the difference between the PDE density $\rho$ and the empirical measure $\hat{\rho}_{h}$ constructed from the self-consistent system, we have for any $s \in[0, t]$

$$
\begin{aligned}
\left\|\left(\rho-\hat{\rho}_{h}\right)(\cdot, s)\right\|^{2}= & \left\|\left(\rho-\hat{\rho}_{h}\right)(\cdot, 0)\right\|^{2}-\int_{0}^{s}\left\|\nabla\left(\rho-\hat{\rho}_{h}\right)(\cdot, q)\right\|^{2} d q \\
& -\int_{0}^{s} \int_{\mathrm{R}^{d}} \nabla \cdot F(x, q)\left(\rho-\hat{\rho}_{h}\right)^{2}(x, q) d x d q \\
& +\operatorname{Tr}(s)+\bar{M}_{s}
\end{aligned}
$$

where $\left\|\left(\rho-\hat{\rho}_{h}\right)(\cdot, 0)\right\|^{2}$ is the initial error and the term of

$$
-\int_{0}^{s}\left\|\nabla\left(\rho-\hat{\rho}_{h}\right)(\cdot, q)\right\|^{2} d q-\int_{0}^{s} \int_{\mathrm{R}^{d}} \nabla \cdot F(x, q)\left(\left(\rho-\hat{\rho}_{h}\right)(x, q)\right)^{2} d x d q
$$


gives the free energy estimation. $\bar{M}_{s}=M_{s}+\tilde{M}_{s}$ is the martingale error from the Ito's formula, where $M_{s}$ is defined by $M_{s}=\sum_{i=1}^{N_{h}} \rho_{0}\left(\theta_{i, h} h\right) M_{s}^{i}$ with

$$
M_{s}^{i}=2 h^{d} \int_{0}^{s} \int_{\mathrm{R}^{d}} \rho(x, q) \nabla \varphi_{\epsilon_{h}}\left(x-\hat{X}_{h, i}(q)\right) d x \cdot d B_{i}(q),
$$

and $\tilde{M}_{s}=\sum_{n=1}^{N_{h}} \rho_{0}\left(\theta_{i, h} h\right) \tilde{M}_{s}^{i}$ with $\tilde{M}_{s}^{i}$ equals to

$$
\begin{aligned}
2 h^{2 d} \int_{0}^{s} \int_{\mathrm{R}^{d}} \varphi_{\epsilon_{h}}(x)\left(\sum_{j=1}^{i-1} \rho_{0}\left(\theta_{j, h} h\right) \nabla \varphi_{\epsilon_{h}}\left(x+\hat{X}_{h, i}(q)-\hat{X}_{h, j}(q)\right)\right. & \\
& \left.-\sum_{j=i+1}^{N_{h}} \rho_{0}\left(\theta_{j, h} h\right) \nabla \varphi_{\epsilon_{h}}\left(x+\hat{X}_{h, j}(q)-\hat{X}_{h, i}(q)\right)\right) d x \cdot d B_{i}(q) .
\end{aligned}
$$

And $\operatorname{Tr}(s)$ is the term of truncation error which is defined as

$$
\begin{aligned}
\operatorname{Tr}(s)= & 2 \int_{0}^{s} \int_{\mathrm{R}^{d}}\left[\sum_{i=1}^{N_{h}} h^{d} \rho_{0}\left(\theta_{i, h} h\right) \varphi_{\epsilon_{h}}\left(x-\hat{X}_{h, i}(q)\right)\left(F(x, q)-F\left(\hat{X}_{h, i}(q), q\right)\right)\right] \cdot \nabla\left(\rho-\hat{\rho}_{h}\right)(x, q) d x d q \\
& +h^{2 d} s\left\|\nabla \varphi_{\epsilon_{h}}\right\|^{2} \sum_{i=1}^{N_{h}} \rho_{0}\left(\theta_{i, h} h\right)^{2} .
\end{aligned}
$$

Proof. To prove the proposition, first note that for any $h$,

$$
\left\|\left(\rho-\rho_{h}\right)(\cdot, s)\right\|^{2}=\|\rho(\cdot, s)\|^{2}-2 \int_{\mathrm{R}^{d}} \rho(x, s) \hat{\rho}_{h}(x, s) d x+\left\|\hat{\rho}_{h}(\cdot, s)\right\|^{2} .
$$

First for the deterministic part of $\|\rho(\cdot, t)\|^{2}$, we have

$$
\begin{aligned}
\int_{\mathrm{R}^{d}} \rho(x, s)^{2} d x & =\int_{\mathrm{R}^{d}} \rho(x, 0)^{2} d x+\int_{0}^{s} \int_{\mathrm{R}^{d}} \rho(x, q)(\Delta \rho(x, q)-2 \nabla \cdot(\rho F)(x, q)) d x d q \\
& =\int_{\mathrm{R}^{d}} \rho(x, 0)^{2} d x-\int_{0}^{s}\|\nabla \rho\|^{2} d q+2 \int_{0}^{s} \int_{\mathrm{R}^{d}} \rho(x, q) F(x, q) \cdot \nabla \rho(x, q) d x d q
\end{aligned}
$$

Then for the second part which equals to

$$
-2 h^{d} \int_{\mathrm{R}^{d}} \rho(x, s) \sum_{i=1}^{N_{h}} \rho_{0}\left(\theta_{i, h} h\right) \varphi_{\epsilon_{h}}\left(x-\hat{X}_{h, i}(s)\right) d x,
$$

note that for each $i$, by Ito's formula, we have

$$
\begin{aligned}
\rho(x, s) \varphi_{\epsilon_{h}}\left(x-\hat{X}_{h, i}(s)\right) & =\rho(x, 0) \varphi_{\epsilon_{h}}\left(x-\hat{X}_{h, i}(0)\right)+\int_{0}^{s} \frac{\partial \rho(x, q)}{\partial t} \varphi_{\epsilon_{h}}\left(x-\hat{X}_{h, i}(q)\right) d q \\
& -\int_{0}^{s} \rho(x, q) \nabla \varphi_{\epsilon_{h}}\left(x-\hat{X}_{h, i}(q)\right) \cdot F\left(\hat{X}_{h, i}(q), q\right) d q \\
& -\int_{0}^{s} \rho(x, q) \nabla \varphi_{\epsilon_{h}}\left(x-\hat{X}_{h, i}(q)\right) \cdot d B_{i}(q) \\
& +\frac{1}{2} \int_{0}^{s} \rho(x, q) \Delta \varphi_{\epsilon_{h}}\left(x-\hat{X}_{h, i}(q)\right) d q .
\end{aligned}
$$

Note that for the second term in the right hand side of the sum above, according to the definition of our PDE,

$$
\int_{0}^{s} \frac{\partial \rho(x, q)}{\partial t} \varphi_{\epsilon_{h}}\left(x-\hat{X}_{h, i}(q)\right) d q=\int_{0}^{s}\left(\frac{1}{2} \Delta \rho(x, q)-\nabla \cdot(\rho(x, q) F(x, q))\right) \varphi_{\epsilon_{h}}\left(x-\hat{X}_{h, i}(q)\right) d q .
$$


Then integrate it over $x \in \mathbb{R}^{d}$, we have

$$
\begin{aligned}
& \int_{0}^{s} \int_{\mathrm{R}^{d}} \frac{\partial \rho(x, q)}{\partial t} \varphi_{\epsilon_{h}}\left(x-\hat{X}_{h, i}(q)\right) d x d q \\
& =-\frac{1}{2} \int_{0}^{s} \int_{\mathrm{R}^{d}} \nabla \rho(x, q) \cdot \nabla \varphi_{\epsilon_{h}}\left(x-\hat{X}_{h, i}(q)\right) d x d q \\
& \quad+\int_{0}^{s} \int_{\mathrm{R}^{d}} \rho(x, q) F(x, q) \cdot \nabla \varphi_{\epsilon_{h}}\left(x-\hat{X}_{h, i}(q)\right) d x d q .
\end{aligned}
$$

Then integrating the third term in (36) over $x \in \mathbb{R}^{d}$ we have by divergence theorem that

$$
\begin{aligned}
-\int_{0}^{s} & \int_{\mathrm{R}^{d}} \rho(x, q) \nabla \varphi_{\epsilon_{h}}\left(x-\hat{X}_{h, i}(q)\right) \cdot F\left(\hat{X}_{h, i}(q), q\right) d x d q \\
& =\int_{0}^{s} \int_{\mathrm{R}^{d}} \varphi_{\epsilon_{h}}\left(x-\hat{X}_{h, i}(q)\right) F\left(\hat{X}_{h, i}(q), q\right) \cdot \nabla \rho(x, q) d x d q .
\end{aligned}
$$

Combining (36), (37) and (38) we have

$$
\begin{aligned}
\int_{\mathrm{R}^{d}} \rho(x, s) \varphi_{\epsilon_{h}}\left(x-\hat{X}_{h, i}(s)\right) d x \\
\quad=\int_{\mathrm{R}^{d}} \rho(x, 0) \varphi_{\epsilon_{h}}\left(x-\hat{X}_{h, i}(0)\right) d x \\
\quad-\int_{0}^{s} \int_{\mathrm{R}^{d}} \nabla \rho(x, q) \cdot \nabla \varphi_{\epsilon_{h}}\left(x-\hat{X}_{h, i}(q)\right) d x d q \\
\quad+\int_{0}^{s} \int_{\mathrm{R}^{d}} \rho(x, q) F(x, q) \cdot \nabla \varphi_{\epsilon_{h}}\left(x-\hat{X}_{h, i}(q)\right) d x d q \\
\quad+\int_{0}^{s} \int_{\mathrm{R}^{d}} \varphi_{\epsilon_{h}}\left(x-\hat{X}_{h, i}(q)\right) F\left(\hat{X}_{h, i}(q), q\right) \cdot \nabla \rho(x, q) d x d q-\frac{h^{-d}}{2} M_{s}^{i}
\end{aligned}
$$

where $M_{s}^{i}$ is a martingale given in (33), i.e.,

$$
M_{s}^{i}=2 h^{d} \int_{0}^{s} \int_{\mathrm{R}^{d}} \rho(x, q) \nabla \varphi_{\epsilon_{h}}\left(x-\hat{X}_{i}(q)\right) d x \cdot d B_{i}(q)
$$

Summing up and taking the weighted average over $i=1,2, \cdots, N_{h}$, we have

$$
\begin{aligned}
-2 \int_{\mathrm{R}^{d}} \hat{\rho}_{h}(x, s) \rho(x, s) d x \\
\quad=-2 \int_{\mathrm{R}^{d}} \hat{\rho}_{h}(x, 0) \rho(x, 0) d x+2 \int_{0}^{s} \int_{\mathrm{R}^{d}} \nabla \rho(x, q) \cdot \nabla \hat{\rho}_{h}(x, q) d x d q \\
\quad-2 \int_{0}^{s} \int_{\mathrm{R}^{d}} \rho(x, q) F(x, q) \cdot \nabla \hat{\rho}_{h}(x, q) d x d q \\
\quad-2 \int_{0}^{s} \int_{\mathrm{R}^{d}} h^{d} \sum_{i=1}^{N_{h}} \rho_{0}\left(\theta_{i, h} h\right) \varphi_{\epsilon_{h}}\left(x-\hat{X}_{h, i}(q)\right) F\left(\hat{X}_{h, i}(q), q\right) \cdot \nabla \rho(x, q) d x d q \\
+M_{s}
\end{aligned}
$$

Lastly, we look at the part of $\left\|\hat{\rho}_{h}(\cdot, s)\right\|^{2}$ which equals to

$$
h^{2 d} \sum_{i, j=1,2, \cdots, N_{h}} \rho_{0}\left(\theta_{i, h} h\right) \rho_{0}\left(\theta_{j, h} h\right) \int_{\mathrm{R}^{d}} \varphi_{\epsilon_{h}}\left(x-\hat{X}_{h, i}(s)\right) \varphi_{\epsilon_{h}}\left(x-\hat{X}_{h, j}(s)\right) d x .
$$


For each $i, j \in\left\{1,2, \cdots, N_{h}\right\}$, if $i=j$, we have directly from change of variables that

$$
\int_{\mathrm{R}^{d}} \varphi_{\epsilon_{h}}\left(x-\hat{X}_{h, i}(s)\right) \varphi_{\epsilon_{h}}\left(x-\hat{X}_{h, i}(s)\right) d x=\left\|\varphi_{\epsilon_{h}}\right\|^{2} .
$$

And if $i \neq j$, say without loss of generality $i<j$ again by change of variables we have

$$
\int_{\mathrm{R}^{d}} \varphi_{\epsilon_{h}}\left(x-\hat{X}_{h, i}(s)\right) \varphi_{\epsilon_{h}}\left(x-\hat{X}_{h, j}(s)\right) d x=\int_{\mathrm{R}^{d}} \varphi_{\epsilon_{h}}(x) \varphi_{\epsilon_{h}}\left(x+\hat{X}_{h, j}(s)-\hat{X}_{h, i}(s)\right) d x .
$$

Then we can again apply the Ito's formula on $\varphi_{\epsilon_{h}}\left(x+\hat{X}_{h, j}(s)-\hat{X}_{h, i}(s)\right)$ and have it equals to:

$$
\begin{aligned}
\varphi_{\epsilon_{h}} & \left(x+\hat{X}_{h, j}(0)-\hat{X}_{h, i}(0)\right)+\int_{0}^{s} \Delta \varphi_{\epsilon_{h}}\left(x+\hat{X}_{h, j}(q)-\hat{X}_{h, i}(q)\right) d q \\
& +\int_{0}^{s} \nabla \varphi_{\epsilon_{h}}\left(x+\hat{X}_{h, j}(q)-\hat{X}_{h, i}(q)\right) \cdot\left(F\left(\hat{X}_{h, j}(q), q\right)-F\left(\hat{X}_{h, i}(q), q\right)\right) d q \\
& +\int_{0}^{s} \nabla \varphi_{\epsilon_{h}}\left(x+\hat{X}_{h, j}(q)-\hat{X}_{h, i}(q)\right) \cdot\left(d B_{j}(q)-d B_{i}(q)\right) .
\end{aligned}
$$

Integrating the first and second terms over $x$, we have

$$
\int_{\mathrm{R}^{d}} \varphi_{\epsilon_{h}}(x) \varphi_{\epsilon_{h}}\left(x+\hat{X}_{h, j}(0)-\hat{X}_{h, i}(0)\right) d x=\int_{\mathrm{R}^{d}} \varphi_{\epsilon_{h}}\left(x-\hat{X}_{h, i}(0)\right) \varphi_{\epsilon_{h}}\left(x-\hat{X}_{h, j}(0)\right) d x
$$

and

$$
\begin{aligned}
\int_{0}^{s} \int_{\mathrm{R}^{d}} \varphi_{\epsilon_{h}}(x) \Delta \varphi_{\epsilon_{h}}\left(x+\hat{X}_{h, j}(q)-\hat{X}_{h, i}(q)\right) d x d q \\
\quad=-\int_{0}^{s} \int_{\mathrm{R}^{d}} \nabla \varphi_{\epsilon_{h}}\left(x-\hat{X}_{h, i}(q)\right) \cdot \nabla \varphi_{\epsilon_{h}}\left(x-\hat{X}_{h, j}(q)\right) d x d q .
\end{aligned}
$$

Moreover, for the third term we have that

$$
\begin{aligned}
\int_{0}^{s} \int_{\mathrm{R}^{d}} \varphi_{\epsilon_{h}}(x) \nabla \varphi_{\epsilon_{h}}\left(x+\hat{X}_{h, j}(q)-\hat{X}_{h, i}(q)\right) \cdot F\left(\hat{X}_{h, j}(q), q\right) d x d q \\
\quad=\int_{0}^{s} \int_{\mathrm{R}^{d}} \varphi_{\epsilon_{h}}\left(x-\hat{X}_{h, j}(q)\right) F\left(\hat{X}_{h, j}(q), q\right) \cdot \nabla \varphi_{\epsilon_{h}}\left(x-\hat{X}_{h, i}(q)\right) d x d q
\end{aligned}
$$

and that

$$
\begin{aligned}
\int_{0}^{s} \int_{\mathrm{R}^{d}} \varphi_{\epsilon_{h}}(x) \nabla \varphi_{\epsilon_{h}}\left(x+\hat{X}_{h, j}(q)-\hat{X}_{h, i}(q)\right) \cdot F\left(X_{h, i}(q), q\right) d x d q \\
\quad=\int_{0}^{s} \int_{\mathrm{R}^{d}} \varphi_{\epsilon_{h}}\left(x-\hat{X}_{h, j}(q)\right) F\left(\hat{X}_{h, i}(q), q\right) \cdot \nabla \varphi_{\epsilon_{h}}\left(x-\hat{X}_{h, i}(q)\right) d x d q \\
=-\int_{0}^{s} \int_{\mathrm{R}^{d}} \varphi_{\epsilon_{h}}\left(x-\hat{X}_{h, i}(q)\right) F\left(\hat{X}_{h, i}(q), q\right) \cdot \nabla \varphi_{\epsilon_{h}}\left(x-\hat{X}_{h, j}(q)\right) d x d q
\end{aligned}
$$

by divergence theorem. We also note that

$$
\int_{\mathrm{R}^{d}} \varphi_{\epsilon_{h}}\left(x-\hat{X}_{h, i}(s)\right) \nabla \varphi_{\epsilon}\left(x-\hat{X}_{h, i}(s)\right) \cdot \vec{F}\left(\hat{X}_{h, i}(s), s\right) d x \equiv 0 .
$$

So after we sum up over all the $i, j$ and have the weighted average, for combinations of the initial values for $i \neq j$ and the constant values for $i=j$ we have

$$
\begin{gathered}
2 \sum_{i<j \in\left\{1,2, \cdots, N_{h}\right\}} h^{2 d} \rho_{0}\left(\theta_{i, h} h\right) \rho_{0}\left(\theta_{j, h} h\right) \int_{\mathrm{R}^{d}} \varphi_{\epsilon_{h}}\left(x-\hat{X}_{h, i}(0)\right) \varphi_{\epsilon_{h}}\left(x-\hat{X}_{h, j}(0)\right) d x \\
+\sum_{i=1}^{N_{h}} h^{2 d} \rho_{0}\left(\theta_{i, h} h\right)^{2}\left\|\varphi_{\epsilon_{h}}\right\|^{2}=\int_{\mathrm{R}^{d}} \hat{\rho}_{h}(x, 0)^{2} d x=\left\|\hat{\rho}_{h}(\cdot, 0)\right\|^{2}
\end{gathered}
$$


Then summing up the integration over $x$ of the second term in (42), we have

$$
\begin{gathered}
-2 \sum_{i<j \in\left\{1,2, \cdots, N_{h}\right\}} h^{2 d} \rho_{0}\left(\theta_{i, h} h\right) \rho_{0}\left(\theta_{j, h} h\right) \int_{0}^{s} \int_{\mathrm{R}^{d}} \nabla \varphi_{\epsilon_{h}}\left(x-\hat{X}_{h, i}(q)\right) \cdot \nabla \varphi_{\epsilon_{h}}\left(x-\hat{X}_{h, j}(q)\right) d x d q \\
=-\int_{0}^{s}\left\|\nabla \hat{\rho}_{h}(\cdot, q)\right\|^{2} d q+h^{2 d} s\left\|\nabla \varphi_{\epsilon_{h}}\right\|^{2} \sum_{i=1}^{N_{h}} \rho_{0}\left(\theta_{i, h} h\right)^{2} .
\end{gathered}
$$

Then summing up the integration over $x$ of the third term in (42) and note that we can add the zero terms in (43) for each $i$ in the weighted average, we have

$$
\begin{array}{r}
2 \sum_{i<j \in\left\{1,2, \cdots, N_{h}\right\}} h^{2 d} \rho_{0}\left(\theta_{i, h} h\right) \rho_{0}\left(\theta_{j, h} h\right) \int_{0}^{s} \int_{\mathrm{R}^{d}} \varphi_{\epsilon_{h}}(x) \nabla \varphi_{\epsilon_{h}}\left(x+\hat{X}_{h, j}(q)-\hat{X}_{h, i}(q)\right) \\
\cdot\left(F\left(\hat{X}_{h, j}(q), q\right)-F\left(\hat{X}_{h, i}(q), q\right)\right) d x d q \\
=2 \int_{0}^{s} \int_{\mathrm{R}^{d}} \sum_{i=1}^{N_{h}} h^{d} \rho_{0}\left(\theta_{i, h} h\right) \varphi_{\epsilon_{h}}\left(x-\hat{X}_{h, i}(q)\right) F\left(\hat{X}_{h, i}(q), q\right) \cdot \nabla \hat{\rho}_{h}(x, q) d x d q
\end{array}
$$

And lastly, if we sum up the last term in (42) for all the $i, j$ 's, we just get $\tilde{M}_{s}=\sum_{n=1}^{N_{h}} \tilde{\rho}_{0}\left(\theta_{i, h} h\right) \tilde{M}_{s}^{i}$ with $\tilde{M}_{s}^{i}$ defined in (34). Thus combining (44)-(46),

$$
\begin{aligned}
\left\|\hat{\rho}_{h}(\cdot, s)\right\|^{2}= & \left\|\hat{\rho}_{h}(\cdot, 0)\right\|^{2}-\int_{0}^{s}\left\|\nabla \hat{\rho}_{h}(\cdot, q)\right\|^{2} d q \\
& +2 \int_{0}^{s} \int_{\mathrm{R}^{d}} \sum_{i=1}^{N_{h}} h^{d} \rho_{0}\left(\theta_{i, h} h\right) \varphi_{\epsilon_{h}}\left(x-\hat{X}_{h, i}(q)\right) F\left(\hat{X}_{h, i}(q), q\right) \cdot \nabla \hat{\rho}_{h}(x, q) d x d q \\
& +\tilde{M}_{s}+h^{2 d} s\left\|\nabla \varphi_{\epsilon_{h}}\right\|^{2} \sum_{i=1}^{N_{h}} \rho_{0}\left(\theta_{i, h} h\right)^{2} .
\end{aligned}
$$

At this point, we can combine (35), (40) and (47) and have

$$
\begin{aligned}
\left\|\left(\rho-\hat{\rho}_{h}\right)(\cdot, s)\right\|^{2} & =\left\|\left(\rho-\hat{\rho}_{h}\right)(\cdot, 0)\right\|^{2}-\int_{0}^{s}\left\|\nabla\left(\rho-\hat{\rho}_{h}\right)(\cdot, q)\right\|^{2} d q+\bar{M}_{s} \\
& +h^{2 d} s\left\|\nabla \varphi_{\epsilon_{h}}\right\|^{2} \sum_{i=1}^{N_{h}} \rho_{0}\left(\theta_{i, h} h\right)^{2} \\
& +2 \int_{0}^{s} \int_{\mathrm{R}^{d}} \sum_{i=1}^{N_{h}} h^{d} \rho_{0}\left(\theta_{i, h} h\right) \varphi_{\epsilon_{h}}\left(x-\hat{X}_{h, i}(q)\right) F\left(\hat{X}_{h, i}(q), q\right) \cdot \nabla \hat{\rho}_{h}(x, q) d x d q \\
& +2 \int_{0}^{s} \int_{\mathrm{R}^{d}} \rho(x, q) F(x, q) \cdot \nabla \rho(x, q) d x d q \\
& -2 \int_{0}^{s} \int_{\mathrm{R}^{d}} \rho(x, q) F(x, q) \cdot \nabla \hat{\rho}_{h}(x, q) d x d q \\
& -2 \int_{0}^{s} \int_{\mathrm{R}^{d}} \sum_{i=1}^{N_{h}} h^{d} \rho_{0}\left(\theta_{i, h} h\right) \varphi_{\epsilon_{h}}\left(x-\hat{X}_{h, i}(q)\right) F\left(\hat{X}_{h, i}(q), q\right) \cdot \nabla \rho(x, q) d x d q .
\end{aligned}
$$

Then plus and minus the term

$$
2 \int_{0}^{s} \int_{\mathrm{R}^{d}} \sum_{i=1}^{N_{h}} h^{d} \rho_{0}\left(\theta_{i, h} h\right) \varphi_{\epsilon_{h}}\left(x-\hat{X}_{h, i}(q)\right) F(x, q) \cdot \nabla\left(\rho-\hat{\rho}_{h}\right)(x, q) d x d q
$$


we have

$$
\begin{aligned}
\left\|\left(\rho-\hat{\rho}_{h}\right)(\cdot, s)\right\|^{2}= & \left\|\left(\rho-\hat{\rho}_{h}\right)(\cdot, 0)\right\|^{2}-\int_{0}^{s}\left\|\nabla\left(\rho-\hat{\rho}_{h}\right)(\cdot, q)\right\|^{2} d q+\operatorname{Tr}(s)+\bar{M}_{s} \\
& +2 \int_{0}^{s} \int_{\mathrm{R}^{d}}\left(\rho-\hat{\rho}_{h}\right)(x, q) F(x, q) \cdot \nabla\left(\rho-\hat{\rho}_{h}\right)(x, q) d x d q .
\end{aligned}
$$

By Green's theorem,

$$
\begin{aligned}
2 \int_{0}^{s} \int_{\mathrm{R}^{d}} & \left(\rho-\hat{\rho}_{h}\right)(x, q) F(x, q) \cdot \nabla\left(\rho-\hat{\rho}_{h}\right)(x, q) d x d q \\
= & -\int_{0}^{s} \int_{\mathrm{R}^{d}} \nabla \cdot F(x, q)\left(\rho-\hat{\rho}_{h}\right)^{2}(x, q) d x d q .
\end{aligned}
$$

We have verified (32) and the proof of Proposition 1 is complete.

\section{Estimation on the Separation}

With Proposition 1 decomposing the distance between $\rho$ and $\hat{\rho}_{h}$ as the sum of several different error terms with different physical and mathematical meanings, we will estimate those error terms one by one. But first, we prove an estimation of separations which shows that, with our initial data, the independent solutions of the self-consistent SDE with high probability cannot be too close to each other. To be specific, we use

$$
E_{j}(t)=\frac{1}{N_{h}} \sum_{i \leq N_{h}: i \neq j} \int_{0}^{t} P\left(\left|\hat{X}_{h, i}(s)-\hat{X}_{h, j}(s)\right| \leq 2 \epsilon_{h}\right) d s
$$

to measure the separation of the self-consistent system. Intuitively, we can see $E_{j}(t)$ as the sum of the average length of time for each particle that is within a distance of $2 \epsilon_{h}$ from particle $j$. And we have the following proposition showing that $E_{j}(t)$ is small, which implies that, with high probability, the path of different particles in the self-consistent system cannot be too close to each other. The reason we want to first prove the proposition can be seen later in (90).

Proposition 2. There exist some constants $C_{1}(t)$ and $C_{2}(t)$ depends only on $t, d$ and $F_{0}$ such that

$$
E_{j}(t) \leq C_{1}(t) \epsilon_{h}^{d-1}+C_{2}(t) \frac{1}{N_{h} \epsilon_{h}}
$$

for all $j=1,2, \cdots, N_{h}$, when $h$ is sufficiently small.

Proof. For any $h$ and $j \leq N_{h}$, Fix $i \neq j, i \leq N_{h}$, and let $\left\{\Omega, \mathcal{F}_{t}^{i, j}, P\right\}$ be our probability measure space where, $\mathcal{F}_{t}^{i, j}$ is the natural filtration generated by $B_{i, j}^{*}(t)=\left[B_{i}(t), B_{j}(t)\right]$, which is a $2 d$-dimensional Brownian motion. Let $\theta_{i, j}(s)=-\left(F\left(\hat{X}_{h, i}(s), s\right), F\left(\hat{X}_{h, j}(s), s\right)\right)$ be the integrand and consider the adapted measurable process

$$
\Gamma_{s}=\int_{0}^{s} \theta_{i, j}(h) \cdot d B_{i, j}^{*}(h)
$$

Note that for any $s \geq 0$,

$$
\left|\theta_{i, j}(s)\right|^{2} \leq 2 d\left\|F_{0}\right\|_{\infty}^{2} .
$$

Thus the Novikov condition (see page 198 of [16] for details) is satisfied, i.e.,

$$
E\left[\exp \left(\frac{1}{2} \int_{0}^{s}\left|\theta_{i, j}(q)\right|^{2} d q\right)\right] \leq \exp \left(s d\left\|F_{0}\right\|_{\infty}^{2}\right)<\infty
$$


by Girsanov Theorem (see Theorem 3.5.1 of [16]) we can define a probability measure $Q$ in our probability space with Radon-Nikodym derivative

$$
\frac{d Q_{i, j}}{d P} \mid \mathcal{F}_{s}=\mathcal{E}_{s}=\exp \left[\Gamma_{s}-\frac{1}{2} \int_{0}^{s}\left|\theta_{i, j}(q)\right|^{2} d q\right]
$$

Then we have

$$
\left[\begin{array}{c}
\hat{X}_{h, i}(s)-\hat{X}_{h, i}(0) \\
\hat{X}_{h, j}(s)-\hat{X}_{h, j}(0)
\end{array}\right]=\left[\begin{array}{c}
B_{i}(s)+\int_{0}^{s} F\left(\hat{X}_{h, i}(q), q\right) d q \\
B_{j}(s)+\int_{0}^{s} F\left(\hat{X}_{h, j}(q), q\right) d q
\end{array}\right]=B_{i, j}^{*}(s)-\left\langle\Gamma, B_{i, j}^{*}\right\rangle_{s}
$$

is a standard $2 d$-dimensional Brownian motion under probability measure $Q_{i, j}$, where $\left\langle\Gamma, B_{i, j}^{*}\right\rangle_{s}$ is again the quadratic covariance between $\Gamma_{s}$ and $B_{i, j}^{*}(s)$. Thus by Radon-Nikodym Theorem we have

$$
\int_{\left|\hat{X}_{h, i}(s)-\hat{X}_{h, j}(s)\right| \leq 2 \epsilon_{h}} \mathcal{E}_{s} d P=P\left(\left|B_{i}(s)-B_{j}(s)+\gamma_{i, j}\right| \leq 2 \epsilon_{h}\right)
$$

where $\gamma_{i, j}=h[\theta(j, h)-\theta(i, h)]$. Moreover,

$$
P\left(\left|\hat{X}_{h, i}(s)-\hat{X}_{h, j}(s)\right| \leq 2 \epsilon_{h}\right) \leq P\left(\mathcal{E}_{s}<\epsilon_{h}^{1 / 2}\right)+P\left(\left|\hat{X}_{h, i}(s)-\hat{X}_{h, j}(s)\right| \leq 2 \epsilon_{h} \cap \mathcal{E}_{s} \geq \epsilon_{h}^{1 / 2}\right)
$$

and for the first part we have,

$$
P\left(\mathcal{E}_{s}<\epsilon_{h}^{1 / 2}\right) \leq P\left(\exp \left[\int_{0}^{s}\left(-\theta_{i, j}(q)\right) \cdot d B_{i, j}^{*}(q)\right]>\epsilon_{h}^{-1 / 2} \exp \left(-s d\left\|F_{0}\right\|_{\infty}^{2}\right)\right) .
$$

To control the right hand side of the inequality above, we consider the $L^{4 d}$ norm:

$$
E\left[\left(\exp \left[\int_{0}^{s}\left(-\theta_{i, j}(q)\right) \cdot d B_{i, j}^{*}(q)\right]\right)^{4 d}\right]=E\left(\exp \left[\int_{0}^{s}\left(-4 d \theta_{i, j}(q)\right) \cdot d B_{i, j}^{*}(q)\right]\right)
$$

and note that again by Girsanov Theorem,

$$
\mathcal{E}_{s}^{\prime}=\exp \left[\int_{0}^{s}\left(-4 d \theta_{i, j}(q)\right) \cdot d B_{i, j}^{*}(q)\right] \exp \left(-8 d^{2} \int_{0}^{s}\left|\theta_{i, j}(q)\right|^{2} d q\right)
$$

is again a Radon-Nikodym derivative. Thus we have

$$
E\left(\mathcal{E}_{s}^{\prime}\right)=1
$$

which combining with (54), implies

$$
E\left(\exp \left[\int_{0}^{s}\left(-4 d \theta_{i, j}(q)\right) \cdot d B_{i, j}^{*}(q)\right]\right) \leq \exp \left(16 d^{3} s\left\|F_{0}\right\|_{\infty}^{2}\right)<\infty .
$$

Combining (57), (59) and Chebyshev's Inequality gives us

$$
P\left(\mathcal{E}_{s}<\epsilon_{h}^{1 / 2}\right) \leq \epsilon_{h}^{2 d} \exp \left(\left(4 d^{2}+16 d^{3}\right) s\left\|F_{0}\right\|_{\infty}^{2}\right) .
$$

Then for the second part, according to (56) we have

$$
\int_{\left|\hat{X}_{h, i}(s)-\hat{X}_{h, j}(s)\right| \leq 2 \epsilon_{h} \cap \mathcal{E}_{s} \geq \epsilon_{h}^{1 / 2}} \mathcal{E}_{s} d P \leq P\left(\left|B_{i}(s)-B_{j}(s)+\gamma_{i, j}\right| \leq 2 \epsilon_{h}\right) .
$$

and thus

$$
\begin{gathered}
P\left(\left|\hat{X}_{h, i}(s)-\hat{X}_{h, j}(s)\right| \leq 2 \epsilon_{h} \cap \mathcal{E}_{s} \geq \epsilon_{h}^{1 / 2}\right) \\
\quad \leq \epsilon_{h}^{-1 / 2} P\left(\left|B_{i}(s)-B_{j}(s)+\gamma_{i, j}\right| \leq 2 \epsilon_{h}\right) .
\end{gathered}
$$


Combining the two inequalities above, we have

$$
\begin{aligned}
P\left(\left|\hat{X}_{h, i}(s)-\hat{X}_{h, j}(s)\right| \leq 2 \epsilon_{h}\right) \leq & \epsilon^{2 d} \exp \left(\left(4 d^{2}+16 d^{3}\right) s\left\|F_{0}\right\|_{\infty}^{2}\right) \\
& +\epsilon_{h}^{-1 / 2} P\left(\left|B_{i}(s)-B_{j}(s)+\gamma_{i, j}\right| \leq 2 \epsilon_{h}\right)
\end{aligned}
$$

for any $s \geq 0$. Integrating (62) on $[0, t]$ and averaging over all $i \neq j, i \leq N_{h}$, we have

$$
\begin{aligned}
E_{j}(t) \leq & \frac{\epsilon_{h}^{2 d}}{\left(4 d^{2}+16 d^{3}\right)\left\|F_{0}\right\|_{\infty}^{2}} \exp \left(\left(4 d^{2}+16 d^{3}\right) t\left\|F_{0}\right\|_{\infty}^{2}\right) \\
& +\frac{1}{N_{h} \epsilon_{h}^{1 / 2}} \sum_{i: i \neq j, i \leq N} \int_{0}^{t} P\left(\left|B_{i}(s)-B_{j}(s)+\gamma_{i, j}\right| \leq 2 \epsilon_{h}\right) d s .
\end{aligned}
$$

According to (63) to proof Proposition 2 it is sufficient to have the following lemma for standard Brownian motions:

Lemma 4.1. For any $t \geq 0$, there is some constant $C_{1}^{*}(t)$ and $C_{2}^{*}(t)$ that depends only on $t$ such that

$$
\frac{1}{N_{h}} \sum_{i: i \neq j, i \leq N_{h}}\left[\int_{0}^{t} P\left(\left|B_{i}(s)-B_{j}(s)+\gamma_{i, j}\right| \leq 2 \epsilon_{h}\right) d s\right] \leq C_{1}^{*}(t) \epsilon_{h}^{d}+C_{2}^{*}(t) \frac{1}{N_{h}} .
$$

Proof. We first note that for any $s$ and $i, j, B_{i}(s)-B_{j}(s)+\gamma_{i, j}$ has a $d$-dimensional normal distribution with mean $\gamma_{i, j}$ and variance $2 s$. So we have

$$
\begin{aligned}
& \int_{0}^{t} P\left(\left|B_{i}(s)-B_{j}(s)+\gamma_{i, j}\right| \leq 2 \epsilon_{h}\right) d s \\
& =\int_{0}^{t} \int_{|x| \leq 2 \epsilon_{h}} \frac{1}{(4 \pi s)^{d / 2}} \exp \left(-\frac{\left|\gamma_{i, j}-x\right|^{2}}{4 s}\right) d x d s \\
& =\int_{|x| \leq 2 \epsilon_{h}} \int_{0}^{t} \frac{1}{(4 \pi s)^{d / 2}} \exp \left(-\frac{\left|\gamma_{i, j}-x\right|^{2}}{4 s}\right) d s d x .
\end{aligned}
$$

To deal with equation (65), we need to separate the case of $d=1, d=2$ and $d \geq 3$.

Case 1: $d=1$. In this case we simply use the bound

$$
\begin{aligned}
& \int_{0}^{t} P\left(\left|B_{i}(s)-B_{j}(s)+\gamma_{i, j}\right| \leq 2 \epsilon_{h}\right) d s \\
& \leq \int_{-2 \epsilon_{h}}^{2 \epsilon_{h}} \int_{0}^{t} s^{-1 / 2} d s d x=8 \epsilon_{h} \sqrt{t} .
\end{aligned}
$$

Averaging over $m$ gives us the desired result.

Case 2: $d=2$. In this case we have

$$
\begin{aligned}
& \int_{0}^{t} P\left(\left|B_{i}(s)-B_{j}(s)+\gamma_{i, j}\right| \leq 2 \epsilon_{h}\right) d s \\
& =\int_{|x| \leq 2 \epsilon_{h}} \int_{0}^{t} \frac{1}{4 \pi s} \exp \left(-\frac{\left|\gamma_{i, j}-x\right|^{2}}{4 s}\right) d s d x .
\end{aligned}
$$

If $\gamma_{i, j} \geq 1$, then for all $\epsilon_{h}<1 / 4$ and $x<2 \epsilon_{h}$ we have

$$
\begin{aligned}
& \int_{|x| \leq 2 \epsilon_{h}} \int_{0}^{t} \frac{1}{4 \pi s} \exp \left(-\frac{\left|\gamma_{i, j}-x\right|^{2}}{4 s}\right) d s d x \\
& \leq \int_{|x| \leq 2 \epsilon_{h}} \int_{0}^{t} \frac{1}{s} \exp \left(-\frac{1}{16 s}\right) d s d x \\
& \leq 16 \epsilon_{h}^{2} \int_{0}^{t} \frac{1}{s} \exp \left(-\frac{1}{16 s}\right) d s
\end{aligned}
$$


When $\gamma_{i, j}<1$ taking $h=\frac{\left|\gamma_{i, j}-x\right|^{2}}{4 s}$, we have

$$
\begin{aligned}
& \int_{0}^{t} P\left(\left|B_{i}(s)-B_{j}(s)+\gamma_{i, j}\right| \leq 2 \epsilon_{h}\right) d s \\
& =\int_{|x| \leq 2 \epsilon_{h}} \int_{0}^{t} \frac{1}{4 \pi s} \exp \left(-\frac{\left|\gamma_{i, j}-x\right|^{2}}{4 s}\right) d s d x \\
& =\int_{|x| \leq 2 \epsilon_{h}} \int_{\left|\gamma_{i, j}-x\right|^{2} / 4 t}^{\infty} \frac{1}{4 \pi h} \exp (-h) d h d x
\end{aligned}
$$

Note that $h^{-1} \exp (-h)<h^{-1}$ and $h^{-1} \exp (-h) \leq \exp (-h)$ when $h \geq 1$. We have

$$
\begin{aligned}
\int_{\left|\gamma_{i, j}-x\right|^{2} / 4 t}^{\infty} h^{-1} \exp (-h) d h & \leq \int_{\left|\gamma_{i, j}-x\right|^{2} / 4 t}^{1} h^{-1} d h+\int_{1}^{\infty} e^{-h} d h \\
& \leq 2\left|\log \left(\left|\gamma_{i, j}-x\right|\right)\right|+|\log t|+1+\log 4
\end{aligned}
$$

Moreover, let $\delta_{1}=C N_{h}^{-1 / 2}$, where $C=L_{D}^{1 / d}, \delta_{2}=\delta_{1}+4 \epsilon_{h}$ and $M=\left[\delta_{2}^{-1}\right]+1$. For all $k=0,1, \cdots, M$ consider the following sets

$$
A_{k}:=\left\{i: k \delta_{2} \leq\left|\gamma_{i, j}\right|<(k+1) \delta_{2}\right\}
$$

By definition, it is easy to see that when $N_{h}$ is large and $\epsilon_{h}$ is small

$$
\bigcup_{k=0}^{M} A_{k} \supset\left\{i:\left|\gamma_{i, j}\right|<1\right\}
$$

If we first look at $A_{0}$, according to that $h \geq C N_{h}^{-1 / 2}$, the little balls $\left\{N\left(\gamma_{i, j}, \delta\right)\right\}_{i \leq N_{h}, i \neq j}$ (where $N(x, y)$ is the neighborhood of $x$ with radius $y)$ have no intersections with each other. And for all $i \in A_{0}$,

$$
N\left(\gamma_{i, j}, \delta_{1}\right) \subset N\left(0, \delta_{1}+\delta_{2}\right)
$$

This immediately implies that

$$
\operatorname{card}\left(A_{0}\right) \leq\left(\frac{\delta_{1}+\delta_{2}}{\delta_{1}}\right)^{2}=\left(2+\frac{4 \epsilon_{h}}{C} N_{h}^{1 / 2}\right)^{2} \leq 8+\frac{32 \epsilon_{h}^{2}}{C^{2}} N_{h}
$$

since the sum of areas of disjoint disks with radius $\delta_{1}$ in $A_{0}$ cannot be larger than the area of $A_{0}$ itself. Thus we have

$$
\frac{1}{N_{h}} \sum_{i \in A_{0}} \int_{0}^{t} P\left(\left|B_{i}(s)-B_{j}(s)+\gamma_{i, j}\right| \leq 2 \epsilon_{h}\right) d s \leq \frac{t}{N_{h}} \operatorname{card}\left(A_{0}\right) \leq \frac{8 t}{N_{h}}+\frac{32 \epsilon_{h}^{2} t}{C^{2}}
$$

Similarly, for each $k \geq 1$ and $i \in A_{k}$,

$$
N\left(\gamma_{i, j}, \delta_{1}\right) \subset\left\{y:(k-1) \delta_{2} \leq|y|<(k+2) \delta_{2}\right\}
$$

which implies that

$$
\operatorname{card}\left(A_{k}\right) \leq \frac{\left[(k+2)^{2}-(k-1)^{2}\right] \delta_{2}^{2}}{\delta_{1}^{2}} \leq 9 k\left(1+\frac{4 \epsilon_{h} N_{h}^{1 / 2}}{C}\right)^{2}
$$

Noting that for all $i \in A_{k}$ and $|x| \leq 2 \epsilon_{h}$

$$
\left|\log \left(\left|\gamma_{i, j}-x\right|\right)\right| \leq \max \left\{\log 2,\left|\log \left(\left|k \delta_{2}-2 \epsilon_{h}\right|\right)\right|\right\} \leq \log 2+\left|\log \left(k \delta_{2}\right)\right| .
$$


Thus according to (67) and the inequality above

$$
\begin{aligned}
& \frac{1}{N_{h}} \sum_{i \in A_{k}} \int_{0}^{t} P\left(\left|B_{i}(s)-B_{j}(s)+\gamma_{i, j}\right| \leq 2 \epsilon_{h}\right) d s \\
& \leq \frac{1}{N_{h}} \sum_{i \in A_{k}}\left[\int_{|x| \leq 2 \epsilon_{h}}|\log t|+\log 4+1+2\left|\log \left(\left|\gamma_{i, j}-x\right|\right)\right| d x\right] \\
& \leq \frac{1}{N_{h}} \sum_{i \in A_{k}}\left[\int_{|x| \leq 2 \epsilon_{h}}|\log t|+\log 16+1+2\left|\log \left(k \delta_{2}\right)\right| d x\right] \\
& \leq\left[\frac{1}{N_{h}} \sum_{i \in A_{k}} 16(|\log t|+\log 16+1) \epsilon_{h}^{2}\right]+\frac{288 \epsilon_{h}^{2} k\left(1+\frac{4 \epsilon_{h} N_{h}^{1 / 2}}{C}\right)^{2}}{N_{h}}\left|\log \left(k \delta_{2}\right)\right| \\
& =\left[\frac{1}{N_{h}} \sum_{i \in A_{k}} 16(|\log t|+\log 16+1) \epsilon_{h}^{2}\right]+\frac{288 \epsilon_{h}^{2}}{C^{2}} \delta_{2}\left[k \delta_{2}\left|\log \left(k \delta_{2}\right)\right|\right]
\end{aligned}
$$

Summing over $k=0,1, \cdots, M$ we have

$$
\begin{aligned}
& \frac{1}{N_{h}} \sum_{i: \gamma_{i, j}<1} \int_{0}^{t} P\left(\left|B_{i}(s)-B_{j}(s)+\gamma_{i, j}\right| \leq 2 \epsilon_{h}\right) d s \\
& \leq \frac{8 t}{N_{h}}+\frac{32 \epsilon_{h}^{2} t}{C^{2}}+16(|\log t|+\log 16+1) \epsilon_{h}^{2}+\frac{288 \epsilon_{h}^{2}}{C^{2}} \sum_{k=1}^{\left[\delta_{2}^{-1}\right]+1} \delta_{2}\left[k \delta_{2}\left|\log \left(k \delta_{2}\right)\right|\right]
\end{aligned}
$$

Note that the last term in the inequality above is a Riemann sum of function $x|\log x|$ and the fact that $x|\log x| \leq \max \left\{\log 2, e^{-1}\right\}<1$ on $[0,2]$.

$$
\sum_{k=1}^{\left[\delta_{2}^{-1}\right]+1} \delta_{2}\left[k \delta_{2}\left|\log \left(k \delta_{2}\right)\right|\right] \leq \int_{0}^{2} d t=2
$$

So we have

$$
\begin{aligned}
& \frac{1}{N_{h}} \sum_{i: \gamma_{i, j}<1} \int_{0}^{t} P\left(\left|B_{i}(s)-B_{j}(s)+\gamma_{i, j}\right| \leq 2 \epsilon_{h}\right) d s \\
& \leq \frac{8 t}{N_{h}}+\frac{32 \epsilon_{h}^{2} t}{C^{2}}+16(|\log t|+\log 16+1) \epsilon_{h}^{2}+\frac{576 \epsilon_{h}^{2}}{C^{2}}
\end{aligned}
$$

Combining (73) and (66), and letting

$$
\begin{aligned}
& C_{1}^{*}(t):=16 \int_{0}^{t} \frac{1}{s} \exp (-1 / 16 s) d s+\frac{32 t}{C^{2}}+16(|\log t|+\log 16+1)+\frac{576}{C^{2}} \\
& C_{2}^{*}(t):=8 t
\end{aligned}
$$

we finally get

$$
\frac{1}{N_{h}} \sum_{i: i \neq j, i \leq N_{h}} \int_{0}^{t} P\left(\left|B_{i}(s)-B_{j}(s)+\gamma_{i, j}\right| \leq 2 \epsilon_{h}\right) d s \leq C_{1}^{*}(t) \epsilon_{h}^{d}+C_{2}^{*}(t) \frac{1}{N_{h}}
$$

when $d=2$, and the proof for case 2 is complete. 
Case 3: $d \geq 3$. The proof in this case is similar but simpler than the case of $d=2$. Again we have

$$
\begin{aligned}
& \int_{0}^{t} P\left(\left|B_{i}(s)-B_{j}(s)+\gamma_{i, j}\right| \leq 2 \epsilon_{h}\right) d s \\
& =\int_{|x| \leq 2 \epsilon_{h}} \int_{0}^{t} \frac{1}{(4 \pi s)^{d / 2}} \exp \left(-\frac{\left|\gamma_{i, j}-x\right|^{2}}{4 s}\right) d s d x .
\end{aligned}
$$

If $\gamma_{i, j} \geq 1$, then for all $\epsilon_{h}<1 / 4$ and $|x|<2 \epsilon_{h}$ we have

$$
\begin{aligned}
& \int_{|x| \leq 2 \epsilon_{h}} \int_{0}^{t} \frac{1}{(4 \pi s)^{d / 2}} \exp \left(-\frac{\left|\gamma_{i, j}-x\right|^{2}}{4 s}\right) d s d x \\
& \leq \int_{|x| \leq 2 \epsilon_{h}} \int_{0}^{t} \frac{1}{s^{d / 2}} \exp \left(-\frac{1}{16 s}\right) d s d x \\
& \leq 2^{2 d} \epsilon_{h}^{d} \int_{0}^{t} \frac{1}{s^{d / 2}} \exp \left(-\frac{1}{16 s}\right) d s
\end{aligned}
$$

When $\gamma_{i, j}<1$ taking $h=\frac{\left|\gamma_{i, j}-x\right|^{2}}{4 s}$, we have

$$
\begin{aligned}
& \int_{0}^{t} P\left(\left|B_{i}(s)-B_{j}(s)+\gamma_{i, j}\right| \leq 2 \epsilon_{h}\right) d s \\
& =\int_{|x| \leq 2 \epsilon_{h}} \int_{0}^{t} \frac{1}{(4 \pi s)^{d / 2}} \exp \left(-\frac{\left|\gamma_{i, j}-x\right|^{2}}{4 s}\right) d s d x \\
& <C_{d} \int_{|x| \leq 2 \epsilon_{h}}\left|\gamma_{i, j}-x\right|^{-d+2} d x .
\end{aligned}
$$

where constant

$$
C_{d}:=2^{4 d} \int_{0}^{\infty} h^{-2+d / 2} \exp (-h) d h .
$$

Then again we can define $\delta_{1}=C N_{h}^{-1 / d}$, where $C=L_{D}^{1 / d}, \delta_{2}=\delta_{1}+4 \epsilon_{h}$ and $M=\left[\delta_{2}^{-1}\right]+1$. For all $k=0,1, \cdots, M$ consider the following sets

$$
A_{k}:=\left\{i: k \delta_{2} \leq\left|\gamma_{i, j}\right|<(k+1) \delta_{2}\right\}
$$

such that

$$
\bigcup_{k=0}^{M} A_{k} \supset\left\{i:\left|\gamma_{i, j}\right|<1\right\}
$$

Then similarly, we have

$$
\operatorname{card}\left(A_{0}\right) \leq\left(\frac{\delta_{1}+\delta_{2}}{\delta_{1}}\right)^{d}=\left(2+\frac{4 \epsilon_{h}}{C} N_{h}^{1 / d}\right)^{d} \leq 2^{2 d-1}+\frac{2^{3 d-1} \epsilon_{h}^{d}}{C^{d}} N_{h}
$$

and

$$
\operatorname{card}\left(A_{k}\right) \leq \frac{\left[(k+2)^{d}-(k-1)^{d}\right] \delta_{2}^{d}}{\delta_{1}^{d}} \leq 3^{d} k^{d-1}\left(1+\frac{4 \epsilon_{h} N_{h}^{1 / d}}{C}\right)^{d}
$$

Thus

$$
\frac{1}{N_{h}} \sum_{i \in A_{0}} \int_{0}^{t} P\left(\left|B_{i}(s)-B_{j}(s)+\gamma_{i, j}\right| \leq 2 \epsilon_{h}\right) d s \leq \frac{t}{N_{h}} \operatorname{card}\left(A_{0}\right) \leq \frac{2^{2 d-1} t}{N_{h}}+\frac{2^{3 d-1} t \epsilon_{h}^{d}}{C^{d}}
$$


and

$$
\begin{aligned}
& \frac{1}{N_{h}} \sum_{i \in A_{k}} \int_{0}^{t} P\left(\left|B_{i}(s)-B_{j}(s)+\gamma_{i, j}\right| \leq 2 \epsilon_{h}\right) d s \\
& \leq \frac{C_{d}}{N_{h}} \sum_{m \in A_{k}} \int_{|x| \leq 2 \epsilon_{h}}\left|\gamma_{i, j}-x\right|^{-d+2} d x \\
& \leq \frac{C_{d}}{N_{h}} 3^{d} k^{d-1}\left(1+\frac{4 \epsilon_{h} N_{h}^{1 / d}}{C}\right)^{d}\left(2^{2 d} \epsilon_{h}^{d}\right) \times\left(2^{d}\left[k\left(C N_{h}^{-1 / d}+2 \epsilon_{h}\right)\right]^{-d+2}\right)
\end{aligned}
$$

Summing over $k=0,1, \cdots, M$,

$$
\begin{aligned}
& \frac{1}{N_{h}} \sum_{i: \gamma_{i, j}<1} \int_{0}^{t} P\left(\left|B_{i}(s)-B_{j}(s)+\gamma_{i, j}\right| \leq 2 \epsilon_{h}\right) d s \\
& \leq \frac{2^{2 d-1} t}{N_{h}}+\frac{2^{3 d-1} t \epsilon_{h}^{d}}{C^{d}}+C_{d}\left(\frac{24}{C}\right)^{d} \epsilon_{h}^{d} \sum_{k=1}^{\left[\delta_{2}^{-1}\right]+1}\left[\left(k \delta_{2}\right) \delta_{2}\right] .
\end{aligned}
$$

Again for the last term we have

$$
\sum_{k=1}^{\left[\delta_{2}^{-1}\right]+1}\left[\left(k \delta_{2}\right) \delta_{2}\right] \leq \int_{0}^{2} t d t=2 .
$$

Thus

$$
\begin{aligned}
& \frac{1}{N_{h}} \sum_{i: \gamma_{i, j}<1} \int_{0}^{t} P\left(\left|B_{i}(s)-B_{j}(s)+\gamma_{i, j}\right| \leq 2 \epsilon_{h}\right) d s \\
& \leq \frac{2^{2 d-1} t}{N_{h}}+\frac{2^{3 d-1} t \epsilon_{h}^{d}}{C^{d}}+2 C_{d}\left(\frac{24}{C}\right)^{d} \epsilon_{h}^{d}
\end{aligned}
$$

Then combining (75) and (81), and letting

$$
\begin{aligned}
& C_{1}^{*}(t):=2^{2 d} \int_{0}^{t} \frac{1}{s^{d / 2}} \exp \left(-\frac{1}{16 s}\right) d s+\frac{2^{3 d-1} t}{C^{d}}+2 C_{d}\left(\frac{24}{C}\right)^{d} \\
& C_{2}^{*}(t):=2^{2 d-1} t
\end{aligned}
$$

We complete the proof of case 3 .

With Lemma 4.1 proved, then according to (63), let

$$
\begin{aligned}
& C_{1}(t)=\frac{1}{\left(4 d^{2}+16 d^{3}\right)\left\|F_{0}\right\|_{\infty}^{2}} \exp \left(\left(4 d^{2}+16 d^{3}\right) t\left\|F_{0}\right\|_{\infty}^{2}\right)+C_{1}^{*}(t) \\
& C_{2}(t)=C_{2}^{*}(t) .
\end{aligned}
$$

Then the proof of Proposition 2 is complete.

\section{Estimation of the Truncation Error}

Back on estimating the errors times, we will first estimate the term $\operatorname{Tr}(s)$ of the truncation error and have the proposition as follows: 
Proposition 3. For $\operatorname{Tr}(s)$ be the truncation error which is defined as

$$
\begin{aligned}
\operatorname{Tr}(s)= & 2 \int_{0}^{s} \int_{\mathrm{R}^{d}}\left[\sum_{i=1}^{N_{h}} h^{d} \rho_{0}\left(\theta_{i, h} h\right) \varphi_{\epsilon_{h}}\left(x-\hat{X}_{h, i}(q)\right)\left(F(x, q)-F\left(\hat{X}_{h, i}(q), q\right)\right)\right] \cdot \nabla\left(\rho-\hat{\rho}_{h}\right)(x, q) d x d q \\
& +h^{2 d} s\left\|\nabla \varphi_{\epsilon_{h}}\right\|^{2} \sum_{i=1}^{N_{h}} \rho_{0}\left(\theta_{i, h} h\right)^{2}
\end{aligned}
$$

in Proposition 1. Then we have when $h$ is sufficiently small,

$$
\begin{aligned}
& E\left(\max \left\{\sup _{s \leq t}\left\{\operatorname{Tr}(s)-\frac{1}{2} \int_{0}^{s}\left\|\nabla\left(\rho-\hat{\rho}_{h}\right)(\cdot, q)\right\|^{2} d q\right\}, 0\right\}\right) \\
& \leq h^{d} t \epsilon_{h}^{-d-2}\|\nabla \varphi\|^{2}\left\|\rho_{0}\right\|_{\infty} U_{D}+2 U_{D}^{2} L_{F}^{2}\|\varphi\|^{2}\left\|\rho_{0}\right\|_{\infty}^{2}\left[\frac{t}{N_{h} \epsilon_{h}^{d-2}}+C_{1}(t) \epsilon_{h}+C_{2}(t) \frac{1}{N_{h} \epsilon_{h}^{d-1}}\right]
\end{aligned}
$$

where $C_{1}$ and $C_{2}$ are the constants in Proposition 2.

Proof. First for the constant term, noting that $\rho_{0}$ is a bounded function and that

$$
\left\|\nabla \varphi_{\epsilon_{h}}\right\|^{2}=\epsilon_{h}^{-2 d-2} \int_{\mathrm{R}^{d}}\left|\nabla \varphi\left(\frac{x}{\epsilon_{h}}\right)\right|^{2} d x=\epsilon_{h}^{-d-2}\|\nabla \varphi\|^{2}
$$

and that $\epsilon_{h}=h^{1 / 4 d}$, we have for any $s \in[0, t]$

$$
s h^{2 d}\left\|\nabla \varphi_{\epsilon_{h}}\right\|^{2} \sum_{i=1}^{N_{h}} \rho_{0}\left(\theta_{i, h} h\right)^{2} \leq t h^{d} \epsilon_{h}^{-d-2}\|\nabla \varphi\|^{2}\left\|\rho_{0}\right\|_{\infty} U_{D}
$$

when $h$ is sufficiently small. Thus, we will concentrate on the non-constant part in the truncation error. By Cauchy Schwarz inequality,

$$
\begin{aligned}
2 \int_{0}^{s} & \int_{\mathrm{R}^{d}}\left[\sum_{i=1}^{N_{h}} h^{d} \rho_{0}\left(\theta_{i, h} h\right) \varphi_{\epsilon_{h}}\left(x-\hat{X}_{h, i}(q)\right)\left(F(x, q)-F\left(\hat{X}_{h, i}(q), q\right)\right)\right] \cdot \nabla\left(\rho-\hat{\rho}_{h}\right)(x, q) d x d q \\
& \leq 2 \int_{0}^{s} \int_{\mathrm{R}^{d}}\left|\sum_{i=1}^{N_{h}} h^{d} \rho_{0}\left(\theta_{i, h} h\right) \varphi_{\epsilon_{h}}\left(x-\hat{X}_{h, i}(q)\right)\left(F(x, q)-F\left(\hat{X}_{h, i}(q), q\right)\right)\right|^{2} d x d q \\
& +\frac{1}{2} \int_{0}^{s}\left\|\nabla\left(\rho-\hat{\rho}_{h}\right)(\cdot, q)\right\|^{2} d q .
\end{aligned}
$$

Let

$$
\operatorname{Res}(s)=\int_{0}^{s} \int_{\mathrm{R}^{d}}\left|\sum_{i=1}^{N_{h}} h^{d} \rho_{0}\left(\theta_{i, h} h\right) \varphi_{\epsilon_{h}}\left(x-\hat{X}_{h, i}(q)\right)\left(F(x, q)-F\left(\hat{X}_{h, i}(q), q\right)\right)\right|^{2} d x d q .
$$

Then by definition in order to show Proposition 2 it is sufficient to prove that

$$
E\left(\sup _{s \leq t} \operatorname{Res}(s)\right) \leq U_{D}^{2} L_{F}^{2}\|\varphi\|^{2}\left\|\rho_{0}\right\|_{\infty}^{2}\left[\frac{t}{N_{h} \epsilon_{h}^{d-2}}+C_{1}(t) \epsilon_{h}+C_{2}(t) \frac{1}{N_{h} \epsilon_{h}^{d-1}}\right]
$$

when $h$ is sufficiently small. To show this, first It is easy to see that we can rewrite the integrand of $\operatorname{Res}(s)$ as

$$
\sum_{i, j=1,2, \cdots, N_{h}} h^{2 d} \rho_{0}\left(\theta_{i, h} h\right) \rho_{0}\left(\theta_{j, h} h\right) R_{i, j}(x, q)
$$


where

$R_{i, j}(x, q)=\varphi_{\epsilon_{h}}\left(x-\hat{X}_{h, i}(q)\right)\left(F(x, q)-F\left(\hat{X}_{h, i}(q), q\right)\right) \cdot \varphi_{\epsilon_{h}}\left(x-\hat{X}_{h, j}(q)\right)\left(F(x, q)-F\left(\hat{X}_{h, j}(q), q\right)\right)$

Note that for any $i, j \leq N, R_{i, j}(x, q) \equiv 0$ when $\left|X_{j}(q)-X_{i}(q)\right|>2 \epsilon_{h}$. And when $\mid X_{i}(q)-$ $X_{j}(q) \mid \leq 2 \epsilon_{h}$, noting that $F$ is Lipschitz continuous with the Lipschitz constant less than or equal to $L_{F}$,

$$
\left|R_{i, j}(x, q)\right| \leq L_{F}^{2} \epsilon_{h}^{2}\left|\varphi_{\epsilon_{h}}\left(x-\hat{X}_{h, i}(q)\right) \varphi_{\epsilon_{h}}\left(x-\hat{X}_{h, j}(q)\right)\right|
$$

Thus for all $i, j \leq N_{h}$, we have the spatial integral

$$
\int_{\mathrm{R}^{d}}\left|R_{i, j}(x, q)\right| d x \leq \epsilon^{2-d} L_{F}^{2}\|\varphi\|^{2} \mathbb{1}_{\left|\hat{X}_{h, i}(q)-\hat{X}_{h, j}(q)\right| \leq 2 \epsilon_{h}} .
$$

Thus we have for any $s \in[0, t]$,

$$
\operatorname{Res}(s) \leq \mathbb{R}^{*}(s)=h^{2 d} \epsilon_{h}^{2-d} L_{F}^{2}\|\varphi\|^{2} \sum_{i, j=1,2, \cdots, N_{h}} \rho_{0}\left(\theta_{i, h} h\right) \rho_{0}\left(\theta_{j, h} h\right) \int_{0}^{s} \mathbb{1}_{\left|\hat{X}_{h, i}(q)-\hat{X}_{h, j}(q)\right| \leq 2 \epsilon_{h}} d q
$$

and note that $\mathbb{R}^{*}(s)$ is monotonically increasing over $s$. Thus to prove Proposition 2 , it suffices to show that

$$
E\left(\mathbb{R}^{*}(t)\right) \leq U_{D}^{2} L_{F}^{2}\|\varphi\|^{2}\left\|\rho_{0}\right\|_{\infty}^{2}\left[\frac{t}{N_{h} \epsilon_{h}^{d-2}}+C_{1}(t) \epsilon_{h}+C_{2}(t) \frac{1}{N_{h} \epsilon_{h}^{d-1}}\right]
$$

when $h$ is sufficiently small. Noting that $\hat{X}_{h, i}(s)-\hat{X}_{h, j}(s)$ is continuous and adaptable to $\mathcal{F}_{t}^{N}$ (which implies progressive), $\mathbb{1}_{\left|\hat{X}_{h, i}(s)-\hat{X}_{h, j}(s)\right| \leq 2 \epsilon_{h}} \times \mathbb{1}_{0 \leq s \leq t}$ is measurable on $[0, t] \times \Omega$ and bounded and thus integrable. By Fubini's Theorem,

$$
\begin{aligned}
E\left[\mathbb{R}^{*}(t)\right] & =h^{2 d} \epsilon_{h}^{2-d} L_{F}^{2}\|\varphi\|^{2}\left(\sum_{i=1: N_{h}} \rho_{0}\left(\theta_{i, h} h\right)^{2} t\right. \\
& \left.+\sum_{i \neq j=1,2, \cdots, N_{h}} \rho_{0}\left(\theta_{i, h} h\right) \rho_{0}\left(\theta_{j, h} h\right) \int_{0}^{t} P\left(\left|\hat{X}_{h, i}(s)-\hat{X}_{h, j}(s)\right| \leq 2 \epsilon_{h}\right) d s\right) \\
& \leq h^{d} U_{D} \epsilon_{h}^{2-d} L_{F}^{2}\|\varphi\|^{2}\left\|\rho_{0}\right\|_{\infty}^{2}\left[t+\sum_{j=1}^{N_{h}} E_{j}(t)\right]
\end{aligned}
$$

where for any $j=1,2, \cdots, N_{h}, E_{j}(t)$ is the separation term defined in (51) in Section 4 . With Proposition 2 proved, then combining (52), (87), (89), and (90) we have the inequality in Proposition 3.

\section{Estimation of the Martingale Error}

In this section, we estimate the martingale error $\bar{M}_{s}=M_{s}+\tilde{M}_{s}$. Out first result is about $M_{s}$ :

Lemma 6.1. For all $s \in[0, t]$, we have the second moment control

$$
E\left(M_{s}^{2}\right) \leq \frac{4 h^{d} U_{D}}{\epsilon^{d+2}}\|\nabla \varphi\|^{2}\left\|\rho_{0}\right\|_{\infty}^{2} \int_{0}^{s}\|\rho(\cdot, q)\|^{2} d q
$$


Proof. Here and in Lemma 6.2, we will use the natural filtration $\mathcal{F}_{s}^{N_{h}}$, which is generated by the Brownian motions $B_{1}(s), \cdots B_{N_{h}}(s)$. Note that $M_{s}=\sum_{i=1}^{N_{h}} \rho_{0}(\theta(i, h) h) M_{s}^{i}$ where

$$
M_{s}^{i}=2 h^{d} \int_{0}^{s} \int_{\mathrm{R}^{d}} \rho(x, q) \nabla \varphi_{\epsilon_{h}}\left(x-\hat{X}_{h, i}(s)\right) d x \cdot d B_{i}(q)=\sum_{k=1}^{d} M_{s}^{i, k},
$$

and

$$
M_{s}^{i, k}=2 h^{d} \int_{0}^{s} \int_{\mathrm{R}^{d}} \rho(x, q) \frac{\partial \varphi_{\epsilon_{h}}\left(x-\hat{X}_{h, i}(q)\right)}{\partial x_{k}} d x d B_{i}^{(k)}(q) .
$$

The $B_{i}^{(k)}(s)$ in the equation above is the $k$ th coordinate of the Brownian motion $B_{i}(s)$ and it is itself a one dimension Brownian motion and a square integrable martingale under filtration $\mathcal{F}_{s}^{N_{h}}$ noting that $B_{i}^{(k)}(s)$ is independent to $B_{j}^{(h)}(s)$ for all $h \neq k$, or $i \neq j$. For each $i$ and $k$ we have the integrand

$$
Y_{i, k}(q)=\int_{\mathrm{R}^{d}} \rho(x, q) \frac{\partial \varphi_{\epsilon_{h}}\left(x-\hat{X}_{h, i}(q)\right)}{\partial x_{k}} d x
$$

continuous and adapted to filtration $\mathcal{F}_{q}^{N}$. Moreover

$$
\left|Y_{i, k}(q)\right|=\left|\int_{\mathrm{R}^{d}} \rho(x, q) \frac{\partial \varphi_{\epsilon_{h}}\left(x-\hat{X}_{h, i}(q)\right)}{\partial x_{k}} d x\right| \leq\left\|\frac{\partial \varphi_{\epsilon_{h}}}{\partial x_{k}}\right\| \times\|\rho(\cdot, s)\|<\infty .
$$

Thus by Theorem 5.2.3 in [8], for all $i \in\{1,2, \cdots, N\}$ and $k \in\{1,2, \cdots, d\}, M_{s}^{i, k}$ is a square integrable martingale with

$$
E\left[\left(M_{s}^{i, k}\right)^{2}\right]=4 h^{2 d} E\left(\int_{0}^{s} Y_{i, k}(q)^{2} d q\right) \leq 4 h^{2 d}\left\|\frac{\partial \varphi_{\epsilon_{h}}}{\partial x_{k}}\right\|^{2} \int_{0}^{t}\|\rho(\cdot, s)\|^{2} d s .
$$

And for all $(i, k) \neq(j, h)$ we have that

$$
\begin{aligned}
\left\langle M_{s}^{i, k}, M_{s}^{j, h}\right\rangle & =\left\langle Y_{i, k} \cdot B_{i}^{(k)}(s), Y_{i, h} \cdot B_{j}^{(h)}(s)\right\rangle \\
& =\int_{0}^{s} Y_{i, k}(s) \cdot Y_{j, h}(q) d\left\langle B_{i}^{(k)}(q), B_{j}^{(h)}(q)\right\rangle \\
& =\int_{0}^{s} Y_{i, k}(s) \cdot Y_{j, h}(q) d 0=0,
\end{aligned}
$$

since $\left\langle B_{i}^{(k)}(s), B_{j}^{(h)}(s)\right\rangle \equiv 0$ for two independent Brownian motions, where $\left\langle X_{s}, Y_{s}\right\rangle$ is the quadratic covariance between the two processes $X_{s}$ and $Y_{s}$, defined by

$$
\left\langle X_{s}, Y_{s}\right\rangle=\frac{1}{2}\left(\left\langle X_{s}+Y_{s}\right\rangle-\left\langle X_{s}\right\rangle-\left\langle Y_{s}\right\rangle\right)
$$

Noting that $M_{s}^{i, k}$ and $M_{s}^{j, h}$ are both square integrable martingales, (93) implies that

$$
E\left(M_{s}^{i, k} M_{s}^{j, h}\right) \equiv 0
$$

Combining (92) and (94) immediately gives us

$$
E\left[\left(M_{s}^{i}\right)^{2}\right]=\sum_{k=1}^{d} E\left[\left(M_{s}^{i, k}\right)^{2}\right] \leq 4 h^{2 d}\left\|\nabla \varphi_{\epsilon_{h}}\right\|^{2} \int_{0}^{s}\|\rho(\cdot, q)\|^{2} d q .
$$


and

$$
E\left(M_{s}^{i} M_{s}^{j}\right)=0
$$

which implies that

$$
E\left(\left(M_{s}\right)^{2}\right)=\sum_{i=1}^{N_{h}} \rho_{0}(\theta(i, h) h)^{2} E\left[\left(M_{t}^{i}\right)^{2}\right] \leq \frac{4 h^{d} U_{D}}{\epsilon^{d+2}}\|\nabla \varphi\|^{2}\left\|\rho_{0}\right\|_{\infty}^{2} \int_{0}^{s}\|\rho(\cdot, q)\|^{2} d q .
$$

Then we estimate the second part of the martingale error and have a lemma as follows:

Lemma 6.2. For all $s \in[0, t]$, we have the second moment control

$$
E\left(\left(\tilde{M}_{s}\right)^{2}\right) \leq \frac{4 h^{d} U_{D}^{3}}{\epsilon^{2 d+2}}\|\varphi\|^{2}\|\nabla \varphi\|^{2}\left\|\rho_{0}\right\|_{\infty}^{4} s .
$$

Proof. Again note that $\tilde{M}_{s}=\sum_{i=1}^{N_{h}} \rho_{0}(\theta(i, h) h) \tilde{M}_{s}^{i}$ with

$$
\tilde{M}_{s}^{i}=\sum_{k=1}^{d} \tilde{M}_{s}^{i, k}
$$

where

$$
\tilde{M}_{s}^{i, k}=\int_{0}^{s} Z_{i, k}(q) d B_{i}^{(k)}(q)
$$

and

$$
\begin{aligned}
Z_{i, k}(q) & =2 h^{2 d} \int_{\mathrm{R}^{d}} \varphi_{\epsilon_{h}}(x) \sum_{j=1}^{i-1} \rho_{0}(\theta(j, h) h) \frac{\partial \varphi_{\epsilon_{h}}\left(x+\hat{X}_{h, i}(q)-\hat{X}_{h, j}(q)\right)}{\partial x_{k}} d x \\
& -2 h^{2 d} \int_{\mathrm{R}^{d}} \varphi_{\epsilon_{h}}(x) \sum_{j=i+1}^{N_{h}} \rho_{0}(\theta(j, h) h) \frac{\partial \varphi_{\epsilon_{h}}\left(x+\hat{X}_{h, i}(q)-\hat{X}_{h, j}(q)\right)}{\partial x_{k}} d x .
\end{aligned}
$$

It is easy to see that the integrand $Z_{i, k}(q)$ is continuous and adapted to $\mathcal{F}_{q}^{N_{h}}$ and that

$$
\left|Z_{i, k}(q)\right| \leq 2 h^{d} U_{D}\left\|\varphi_{\epsilon_{h}}\right\| \cdot\left\|\frac{\partial \varphi_{\epsilon_{h}}}{\partial x_{k}}\right\| \cdot\left\|\rho_{0}\right\|_{\infty}
$$

Then again accordion to Theorem 5.2.3 in [8] we have for all $i \in\left\{1,2, \cdots, N_{h}\right\}$ and $k \in$ $\{1,2, \cdots, d\}, M_{s}^{i, k}$ is a square integrable martingale with that

$$
E\left[\left(\tilde{M}_{s}^{i, k}\right)^{2}\right]=E\left(\int_{0}^{s} Z_{i, k}(q)^{2} d q\right) \leq 4 h^{2 d} U_{D}^{2}\left\|\varphi_{\epsilon_{h}}\right\|^{2} \cdot\left\|\frac{\partial \varphi_{\epsilon_{h}}}{\partial x_{k}}\right\|^{2} \cdot\left\|\rho_{0}\right\|_{\infty}^{2} s .
$$

and that for all $(i, k) \neq(j, h)$ we have that

$$
\begin{aligned}
\left\langle\tilde{M}_{s}^{i, k}, \tilde{M}_{s}^{j, h}\right\rangle & =\left\langle Z_{i, k} \cdot B_{i}^{(k)}(s), Z_{i, h} \cdot B_{j}^{(h)}(s)\right\rangle \\
& =\int_{0}^{s} Z_{i, k}(q) \cdot Z_{j, h}(q) d\left\langle B_{i}^{(k)}(q), B_{j}^{(h)}(q)\right\rangle \\
& =\int_{0}^{s} Z_{i, k}(q) \cdot Z_{j, h}(q) d 0=0,
\end{aligned}
$$


which implies that

$$
E\left(\tilde{M}_{s}^{i, k} \tilde{M}_{s}^{j, h}\right) \equiv 0
$$

Thus we immediately have

$$
E\left[\left(\tilde{M}_{s}^{i}\right)^{2}\right] \leq 4 h^{2 d} U_{D}^{2}\left\|\varphi_{\epsilon_{h}}\right\|^{2} \cdot\left\|\nabla \varphi_{\epsilon_{h}}\right\|^{2} \cdot\left\|\rho_{0}\right\|_{\infty}^{2} s
$$

and $E\left(\tilde{M}_{s}^{i} \tilde{M}_{s}^{j}\right)=0$ for all $i \neq j$. Thus

$$
E\left(\left(\tilde{M}_{s}\right)^{2}\right)=\sum_{i=1}^{N_{h}} \rho_{0}(\theta(i, h) h)^{2} E\left(\left(\tilde{M}_{s}^{i}\right)^{2}\right) \leq \frac{4 h^{d} U_{D}^{3}}{\epsilon^{2 d+2}}\|\varphi\|^{2}\|\nabla \varphi\|^{2}\left\|\rho_{0}\right\|_{\infty}^{4} s .
$$

\section{Estimation of the Initial Error}

To estimate the distance between the initial empirical density

$$
\rho_{h}(x, 0)=\sum_{i=1}^{N_{h}} h^{d} \rho_{0}\left(\theta_{i, h}\right) \varphi_{\epsilon_{h}}\left(x-\theta_{i, h}\right) .
$$

and $\rho_{0}(x)$, we have a lemma as follows:

Lemma 7.1. For Sufficiently small $h$,

$$
\left\|\rho_{h}(x, 0)-\rho_{0}(x)\right\|^{2} \leq \epsilon_{h}^{1 / 2} .
$$

Proof. To prove this lemma, we introduce the following intermediate density function

$$
\tilde{\rho}_{h}(x, 0)=\rho_{0}(x)\left[\sum_{i=1}^{N_{h}} h^{d} \varphi_{\epsilon_{h}}\left(x-\theta_{i, h}\right)\right] .
$$

To estimate the distance between $\tilde{\rho}_{h}(x, 0)$ and $\rho_{h}(x, 0)$, we have for any $x \in D_{1}$,

$$
\begin{aligned}
\left|\rho_{h}(x, 0)-\tilde{\rho}_{h}(x, 0)\right| & \leq \sum_{i=1}^{N_{h}} h^{d}\left|\rho_{0}\left(\theta_{i, h}\right)-\rho_{0}(x)\right| \varphi_{\epsilon_{h}}\left(x-\theta_{i, h}\right) \\
& =\sum_{\left|x-\theta_{i, h}\right|_{\infty} \leq \epsilon_{h}} h^{d}\left|\rho_{0}\left(\theta_{i, h}\right)-\rho_{0}(x)\right| \varphi_{\epsilon_{h}}\left(x-\theta_{i, h}\right)
\end{aligned}
$$

Noting that $\rho_{0}$ is a Lipschitz continuous function with Lipschitz constant $L_{\rho_{0}}$, and that

$$
\varphi_{\epsilon_{h}}(x)=\frac{1}{\epsilon_{h}^{d}} \varphi\left(\frac{x}{\epsilon_{h}}\right)
$$

we have

$$
\begin{aligned}
\left|\rho_{h}(x, 0)-\tilde{\rho}_{h}(x, 0)\right| & \leq \sum_{\left|x-\theta_{i, h}\right| \leq \epsilon_{h}} h^{d}\left|\rho_{0}\left(\theta_{i, h}\right)-\rho_{0}(x)\right| \varphi_{\epsilon_{h}}\left(x-\theta_{i, h}\right) \\
& \leq L_{\rho_{0}}\|\varphi\|_{\infty} \sum_{\left|x-\theta_{i, h}\right|_{\infty} \leq \epsilon_{h}} \frac{h^{d}}{\epsilon_{h}^{d-1}} .
\end{aligned}
$$


Recalling by definition $\epsilon_{h}=h^{1 / 6 d} \gg h$, when $h$ is sufficiently small, we have

$$
\left|\rho_{h}(x, 0)-\tilde{\rho}_{h}(x, 0)\right| \leq L_{\rho_{0}}\|\varphi\|_{\infty} 3^{d} \epsilon_{h} .
$$

And for any $x \in D_{1}^{c}$

$$
\tilde{\rho}_{h}(x, 0)=\rho_{h}(x, 0)=0 .
$$

And for the distance between $\tilde{\rho}_{h}(x, 0)$ and $\rho_{0}(x)$, we first note that for any $x \in D$

$$
\tilde{\rho}_{h}(x, 0)=\rho_{0}(x)\left[\sum_{i \leq N_{h},\left|x-\theta_{i, h}\right| \leq \epsilon_{h}} h^{d} \varphi_{\epsilon_{h}}\left(x-\theta_{i, h}\right)\right] .
$$

Then for any $x \in D$ and $i$ such that $\left|x-\theta_{i, h}\right| \leq \epsilon_{h}$,

$$
\left|h^{d} \varphi_{\epsilon_{h}}\left(x-\theta_{i, h}\right)-\int_{C\left(x-\theta_{i, h}, h\right)} \varphi_{\epsilon_{h}}(y) d y\right| \leq h^{d+1} \epsilon_{h}^{-d-1}\|\nabla \varphi\|_{\infty} .
$$

Summing up over all such neighborhood of size $h$ centered at $x-\theta_{i, h}$, we have

$$
\begin{aligned}
\tilde{\rho}_{h}(x, 0) & \geq \rho_{0}(x)\left[\int_{|x-y|_{\infty} \leq \epsilon_{h} \cap\{y \in D\}} \varphi_{\epsilon_{h}}(x-y) d y-\int_{|x-y|_{\infty} \leq 2 \epsilon_{h}} h \epsilon_{h}^{-d-1}\|\nabla \varphi\|_{\infty} d y\right] \\
& =\rho_{0}(x)\left[\int_{|x-y|_{\infty} \leq \epsilon_{h} \cap\{y \in D\}} \varphi_{\epsilon_{h}}(x-y) d y-4^{d} h \epsilon_{h}^{-1}\|\nabla \varphi\|_{\infty}\right],
\end{aligned}
$$

and

$$
\begin{aligned}
\tilde{\rho}_{h}(x, 0) & \leq \rho_{0}(x)\left[\int_{|x-y|_{\infty} \leq 2 \epsilon_{h}} \varphi_{\epsilon_{h}}(x-y) d y+\int_{|x-y|_{\infty} \leq 2 \epsilon_{h}} h \epsilon_{h}^{-d-1}\|\nabla \varphi\|_{\infty} d y\right] \\
& =\rho_{0}(x)\left[1+4^{d} h \epsilon_{h}^{-1}\|\nabla \varphi\|_{\infty}\right],
\end{aligned}
$$

Thus for any $x$ such that $d\left(x, D^{c}\right)>\epsilon_{h}$, noting that $|x-y|_{\infty} \leq \epsilon_{h} \subset\{y \in D\}$, we have

$$
\left|\tilde{\rho}_{h}(x, 0)-\rho_{0}(x)\right| \leq 4^{d} h \epsilon_{h}^{-1}\|\nabla \varphi\|_{\infty}\left\|\rho_{0}\right\|_{\infty}=4^{d} \epsilon_{h}^{5 d-1}\|\nabla \varphi\|_{\infty}\left\|\rho_{0}\right\|_{\infty}
$$

And for any $x$ such that $d\left(x, D^{c}\right) \leq \epsilon_{h}$, we have

$$
0 \leq \rho_{0}(x) \leq L_{\rho_{0}} \epsilon_{h},
$$

and

$$
0 \leq \tilde{\rho}_{h}(x, 0) \leq \rho_{0}(x)\left[1+4^{d} h \epsilon_{h}^{-1}\|\nabla \varphi\|_{\infty}\right]
$$

Thus

$$
\left|\tilde{\rho}_{h}(x, 0)-\rho_{0}(x)\right| \leq L_{\rho_{0}} \epsilon_{h}\left[1+4^{d} h \epsilon_{h}^{-1}\|\nabla \varphi\|_{\infty}\right]
$$

Combining (105) and (106), we have that when $h$ is sufficiently small, for any $x \in D$

$$
\left|\tilde{\rho}_{h}(x, 0)-\rho_{0}(x)\right| \leq 2 L_{\rho_{0}} \epsilon_{h} .
$$

And for any $x \in D^{c}$

$$
\tilde{\rho}_{h}(x, 0)=\rho_{0}(x)=0 .
$$

Thus combining (104) and (107), we have for any $x \in D_{1}$,

$$
\left|\rho_{h}(x, 0)-\rho_{0}(x)\right| \leq L_{\rho_{0}}\left(2+3^{d}\|\varphi\|_{\infty}\right) \epsilon_{h} .
$$


and for any $x \in D_{1}^{c}$

$$
\rho_{h}(x, 0)=\rho_{0}(x)=0 .
$$

Thus

$$
\begin{aligned}
\left\|\rho_{h}(x, 0)-\rho_{0}(x)\right\|^{2} & =\int_{D_{1}}\left|\rho_{h}(x, 0)-\rho_{0}(x)\right|^{2} d x \\
& \leq U_{D} L_{\rho_{0}}^{2}\left(2+3^{d}\|\varphi\|_{\infty}\right)^{2} \epsilon_{h}^{2} \leq \epsilon_{h}^{1 / 2} .
\end{aligned}
$$

The Proof of Lemma 7.1 is complete.

\section{Proof of Theorem 2 and Theorem 1}

In this section, we will put all the estimations we have on different error terms together to finish the proof of Theorem 2 and then Theorem 1. First since that in (84) we have

$$
\begin{aligned}
& E\left(\max \left\{\sup _{s \leq t}\left\{\operatorname{Tr}(s)-\frac{1}{2} \int_{0}^{s}\left\|\nabla\left(\rho-\hat{\rho}_{h}\right)(\cdot, q)\right\|^{2} d q\right\}, 0\right\}\right) \\
& \leq h^{d} t \epsilon_{h}^{-d-2}\|\nabla \varphi\|^{2}\left\|\rho_{0}\right\|_{\infty} U_{D}+2 U_{D}^{2} L_{F}^{2}\|\varphi\|^{2}\left\|\rho_{0}\right\|_{\infty}^{2}\left[\frac{t}{N_{h} \epsilon_{h}^{d-2}}+C_{1}(t) \epsilon_{h}+C_{2}(t) \frac{1}{N_{h} \epsilon_{h}^{d-1}}\right] \\
& \leq C_{\text {truncate }}^{2}(t) \epsilon_{h}
\end{aligned}
$$

since that $\epsilon_{h}=h^{1 / 6 d}$, where

$$
C_{\text {truncate }}(t)=\sqrt{\|\nabla \varphi\|^{2}\left\|\rho_{0}\right\|_{\infty} U_{D} t+2 U_{D}^{2} L_{F}^{2}\|\varphi\|^{2}\left\|\rho_{0}\right\|_{\infty}^{2}\left[L_{D} t+C_{1}(t)+C_{2}(t)\right]} .
$$

Thus according to Chebyshev inequality, let event

$$
A_{T}=\left\{\sup _{s \leq t}\left\{\operatorname{Tr}(s)-\frac{1}{2} \int_{0}^{s}\left\|\nabla\left(\rho-\hat{\rho}_{h}\right)(\cdot, q)\right\|^{2} d q\right\} \leq C_{\text {truncate }}(t) \epsilon_{h}^{1 / 2}\right\} .
$$

we have $P\left(A_{T}\right) \geq 1-C_{\text {truncate }}(t) \epsilon_{h}^{1 / 2}$.

Similarly, in Lemma 6.1 we have that $M_{s}$ is a $L^{2}$ integrable martingale and for any $s \leq t$,

$$
E\left(M_{s}^{2}\right) \leq \frac{4 h^{d} U_{D}}{\epsilon^{d+2}}\|\nabla \varphi\|^{2}\left\|\rho_{0}\right\|_{\infty}^{2} \int_{0}^{s}\|\rho(\cdot, q)\|^{2} d q .
$$

Since $F$ is Lipschitz continuous from $\mathbb{R}^{d} \rightarrow \mathbb{R}^{d}$, it is also differentiable almost everywhere by Rademacher's theorem, see Theorem 3.1.6 of [9]. Thus for any $x$ such that $F$ is differentiable, we have $\|\nabla \cdot \vec{F}\|_{L^{\infty}} \leq d L_{F}$, which, according to (35), implies that $\|\rho(\cdot, s)\|^{2} \leq e^{C_{0} s}\left\|\rho_{0}\right\|^{2}$ where $C_{0}=2 d L_{F} \geq 2\|\nabla \cdot \vec{F}\|_{L^{\infty}}$. So we have

$$
E\left(M_{t}^{2}\right) \leq \frac{4 h^{d} U_{D} e^{C_{0} t}}{\epsilon_{h}^{d+2} C_{0}}\|\nabla \varphi\|^{2}\left\|\rho_{0}\right\|_{\infty}^{2} \leq \frac{4 U_{D} e^{C_{0} t}}{C_{0}}\|\nabla \varphi\|^{2}\left\|\rho_{0}\right\|_{\infty}^{2} \epsilon_{h}^{3}
$$

since $\epsilon_{h}=h^{1 / 6 d}$. By Doob and Chebyshev inequality, letting

$$
C_{M}(t)=\left(\frac{16 U_{D} e^{C_{0} t}}{C_{0}}\|\nabla \varphi\|^{2}\left\|\rho_{0}\right\|_{\infty}^{2}\right)^{1 / 3}
$$

and event

$$
A_{M}=\left\{\sup _{s \in[0, t]}\left|M_{s}\right| \leq C_{M}(t) \epsilon_{h}\right\}
$$


we have $P\left(A_{M}\right) \geq 1-C_{M}(t) \epsilon_{h}$. Similarly, according to Lemma $6.2, \tilde{M}_{s}$ is a $L^{2}$ integrable martingale and

$$
E\left(\left(\tilde{M}_{t}\right)^{2}\right) \leq \frac{4 h^{d} U_{D}^{3}}{\epsilon_{h}^{2 d+2}}\|\varphi\|^{2}\|\nabla \varphi\|^{2}\left\|\rho_{0}\right\|_{\infty}^{4} t \leq 4 U_{D}^{3}\|\varphi\|^{2}\|\nabla \varphi\|^{2}\left\|\rho_{0}\right\|_{\infty}^{4} t \epsilon_{h}^{2}
$$

for $\epsilon_{h}=h^{1 / 6 d}$. Then let

$$
C_{\tilde{M}}(t)=\left(16 U_{D}^{3}\|\varphi\|^{2}\|\nabla \varphi\|^{2}\left\|\rho_{0}\right\|_{\infty}^{4} t\right)^{1 / 3}
$$

and event

$$
A_{\tilde{M}}=\left\{\sup _{s \in[0, t]}\left|\tilde{M}_{s}\right| \leq C_{\tilde{M}}(t) \epsilon_{h}^{2 / 3}\right\} .
$$

We have again by Doob and Chebyshev inequality, $P\left(A_{\tilde{M}}\right) \geq 1-C_{\tilde{M}}(t) \epsilon_{h}^{2 / 3}$. Finally note that in Lemma 7.1, the initial error is bounded by

$$
\left\|\rho_{0}-\hat{\rho}_{h}(\cdot, 0)\right\| \leq \epsilon_{h}^{1 / 2}
$$

when $h$ is sufficiently small. Then under the event

$$
A=A_{T} \cap A_{M} \cap A_{\tilde{M}}
$$

such that

$$
\begin{aligned}
P(A) & \geq 1-C_{\text {truncate }}(t) \epsilon_{h}^{1 / 2}-C_{M}(t) \epsilon_{h}-C_{\tilde{M}}(t) \epsilon_{h}^{2 / 3} \\
& \geq 1-\left[C_{\text {truncate }}(t)+C_{M}(t)+C_{\tilde{M}}(t)\right] \epsilon_{h}^{1 / 2}
\end{aligned}
$$

we have for any $s \in[0, t]$,

$$
\begin{aligned}
& \left\|\left(\rho-\hat{\rho}_{h}\right)(\cdot, s)\right\|^{2}+\frac{1}{2} \int_{0}^{s}\left\|\nabla\left(\rho-\hat{\rho}_{h}\right)(\cdot, q)\right\|^{2} d q \\
& \leq \epsilon_{h}^{1 / 2}-\int_{0}^{s} \int_{\mathrm{R}^{d}} \nabla \cdot F(x, q)\left[\rho(x, q)-\hat{\rho}_{h}(x, q)\right]^{2} d x d q+\left[C_{\text {truncate }}(t)+C_{M}(t)+C_{\tilde{M}}(t)\right] \epsilon_{h}^{1 / 2} .
\end{aligned}
$$

Noting that the inequality above holds for all $s \in[0, t]$ and that $\epsilon_{h}^{1 / 2}=h^{1 / 12 d}$, then let

$$
c_{1}(t)=2 e^{C_{0} t}\left[1+C_{\text {truncate }}(t)+C_{M}(t)+C_{\tilde{M}}(t)\right] .
$$

Gronwall's inequality finishes the proof of Theorem 2.

With Theorem 2 proved, combining it with the result of Theorem 3 and let

$$
c(t)=c_{0}(t)+c_{1}(t)
$$

The proof of Theorem 1 is complete. 


\section{References}

[1] F. Bolley, J. A. Cañizo and J. A. Carrillo, Mean-field limit for the stochastic Vicsek model, Applied Mathematics Letters, 25 (2012), 339-343.

[2] F. Bolley, J. A. Cañizo and J. A. Carrillo, Stochastic mean-field limit: non-Lipschitz forces and swarming, Math. Models Methods Appl. Sci. 21 (2011), 2179-2210.

[3] F. Bolley, A. Guillin and C. Villani, Quantitative concentration inequalities for empirical measures on non-compact spaces, Prob. Theory Rel. Fields, 137 (2007), 541-593.

[4] P. Calderoni and M. Pulvirenti, Propagation of chaos for Burgers' equation, Ann. Inst. H. Poincare, Sec. A (N.S.), 39 (1983), 85-97.

[5] A. J. Chorin, Numerical study of slightly viscous flow, J. Fluid Mech., 57 (1973), 785-796.

[6] G. H. Cottet, P. Koumoutsakos, Vortex Methods: Theory and Practice, Cambridge University Press, 2000.

[7] K. Craig, A. Bertozzi, A Blob Method for the Aggregation Equation, Math. Comp., to appear

[8] S. N. Ethier and R. G. Kurtz, Markov Processes: Characterization and Convergence, John Wiley \& Sons, Inc. 2005

[9] H. Federer, Geometric Measure Theory, Springer-Verlag, New York, 1969.

[10] J. Fontbona, Stochastic vortex method for forced three-dimensional Navier-Stokes equations and pathwise convergence rate, Ann. Appl. Probab., 20 (2010), 1761-1800.

[11] N. Fournier, M. Hauray and S. Mischler, Propagation of chaos for the 2D viscous vortex model, arXiv:1212.1437.

[12] J. Goodman, Convergence of the random vortex method, Communications on Pure and Applied Mathematics, 40 (1987) 189-220.

[13] E. Gutkin and M. Kac, Propagation of chaos and the Burgers? equation, SIAM J. Appl. Math., 43 (1983), 971-980.

[14] H. Huang and J.-G. Liu, Error estimate of a random particle blob method for the KellerSegel equation, preprint.

[15] M. Kac, Foundation of kinetic theory, Proc. Third Berkeley Symp., Math. Stat. and Probab., 3, Univ. of Calif., 1956, 171-197.

[16] I. Karatzas and S. Shreve, Brownian Motion and Stochastic Calculus, 2nd edition Springer, 1991

[17] P. Koumoutsakos and A. Leonard, High-resolution simulations of the flow around an impulsively started cylinder using vortex methods, J. Fluid Mech. 96, (1995) 1-38.

[18] J.-G. Liu and R. Yang, Propagation of chaos for Keller-Segel equation, preprint.

[19] J.-G. Liu and R. Yang, A random particle blob method for the Keller-Segel equation and convergence analysis, preprint.

[20] J.-G. Liu and R. Yang, Propagation of chaos for large Brownian particle systems with Coulomb interaction, preprint.

[21] J.-G. Liu and Y. Zhang, Convergence of diffusion-drift many particle systems in probability under a Sobolev norm. Proceedings of Particle Systems and Partial Differential Equations - III, 2015, to appear.

[22] D.-G. Long, Convergence of the random vortex method in two dimensions, Journal of the American Mathematical Society, 1(1988), 779-804. 
[23] C. Marchioro and M. Pulvirenti, Hydrodynamics in two dimensions and vortex theory, Com- mun. Math. Phys., 84 (1982), 483-504.

[24] H. P. McKean, Propagation of chaos for a class of non-linear parabolic equation, Lecture Series in Differential Equations, session 7, Catholic Univ., 1967, 177-194.

[25] H. Osada, Propagation of chaos for the two dimensional Navier-Stokes equation, Probabilistic methods in mathematical physics (Katata/Kyoto, 1985), 303-334, Academic Press, Boston, MA, 1987.

[26] F. Rezakhanlou, Kinetic limits for a class of interacting particle systems, Probab. Theory Related Fields, 104 (1996), 97-146.

[27] F. Rezakhanlou, and J. Tarver, Boltzmann-Grad limit for a particle system in continuum, Annales de l'Institut Henri Poincarré Probab. Stat., 33 (1997), 753-796.

[28] T. Shiga and H. Tanaka, Central limit theorem for a Markovian system of particles in mean-field interaction, Zeitschrift für Wahrscheinlichkeitstheorie und verwandte Gebiete, 69 (1985), 439-459.

[29] A. Stevens, The derivation of chemotaxis equations as limit dynamics of moderately interacting stochastic many-particle systems, SIAM J. Appl. Math., 61 (2000), 183-212.

[30] A. S. Sznitman, Topics in propagation of chaos, In Ecole d'Eté de Probabilités de SaintFlour XIX-1989, Lecture Notes in Math. 1464. Springer, Berlin, 1991.

[31] A. S. Sznitman, A propagation of chaos result for Burgers' equation, Probab. Theory Related Fields, 71 (1986), 581-613. 